\title{
PARTicle Clustering AND COATING ACROSS SCALES: MICROFLUIDIC AND MACROFLUIDIC EXPERIMENTS
}

\author{
by
}

\author{
Steven G. Jones \\ Bachelor of Engineering, Ryerson University, 2013
}

\author{
A thesis \\ presented to Ryerson University \\ in partial fulfillment of the \\ requirements for the degree of \\ Master of Applied Science \\ in the Program of \\ Mechanical and Industrial Engineering
}

Toronto, Ontario, Canada, 2015

(C)Steven G. Jones 2015 


\section{AUTHOR'S DECLARATION FOR ELECTRONIC SUBMISSION OF A THESIS}

I hereby declare that I am the sole author of this thesis. This is a true copy of the thesis, including any required final revisions, as accepted by my examiners.

I authorize Ryerson University to lend this thesis to other institutions or individuals for the purpose of scholarly research.

I further authorize Ryerson University to reproduce this thesis by photocopying or by other means, in total or in part, at the request of other institutions or individuals for the purpose of scholarly research.

I understand that my thesis may be made electronically available to the public. 


\title{
Particle clustering and coating across scales: microfluidic and macrofluidic experiments
}

\author{
Master of Applied Science 2015 \\ Steven G. Jones \\ Mechanical and Industrial Engineering \\ Ryerson University
}

\begin{abstract}
In this thesis, I study the self-assembly of monodisperse colloidal particles on liquid-liquid interfaces. Specifically, I examine the relevant parameters that govern the size of self-assembled clusters when they pass through a liquid-liquid interface. I first describe a millimeter length-scale self-assembly system, where I find that the number of particles within a sinking cluster is proportional to a power law of the dimensionless Bond number. I find that the sphere deposition geometry also plays an important role, where I observe distinctly different scaling for monolayer rafts in comparison to stacked sphere clusters. I then develop an analogous microfluidic self-assembly system, where I use a magnetic field gradient to self-assemble paramagnetic microparticles on an aqueous two-phase liquid-liquid interface. Here, I observe empirically that the number of particles within a microparticle cluster scales inversely with the magnetic Bond number.
\end{abstract}




\section{Acknowledgements}

First and foremost, I would like to thank my supervisor, mentor, and friend Dr. Scott S. H. Tsai for his enormous contribution to my exceptionally positive graduate studies experience. Scott's extraordinary enthusiasm for science and discovery, and his appreciation for classical writing and applied mathematics is both inspiring and contagious.

I also gratefully acknowledge the contributions of Dr. Byeong-Ui Moon and Niki Abbasi, who both played a crucial role in the development of these self-assembly systems. Specifically I would like to thank Byeong-Ui for his expertise in experimental microfluidics and microfabrication, and I'd like to thank Niki Abbasi for her help preparing and running countless experiments. Without Niki and Byeong-Ui this project would not have been possible.

I would also like to acknowledge the positive influence of the numerous students and technical staff who were involved at various stages of this project. In particular, I would like to thank Vaskar Gnyawali and Devin Ostrom for their assistance with 3D printing, Lindsey Fiddes for her expertise with microfabrication, and Elizabeth Berndl and Sasha Cai Lesher-Perez for their help with cell culturing.

Moreover, I would like to thank the dozens of other passionate graduate students with whom I have had the privilege of working with. Brian Battaglia, Nevetha Yogarajah, Vaskar Gnyawali, Huma Inayat, Abhinav Ahuja, Vivian Truong and Farbod Ahmadzadeh of the Tsai Lab, Adel Alhalawan and Omar Rodriguez of the Towler Lab, Lianette Rivera, Sasha Cai Lesher-Perez, Joseph Labuz, Cedric Bathany and Brendan Leung of the Takayama lab, as well as numerous others at University of Michigan, the University of Toronto, and Ryerson University. Thank you all for your thoughtful discussions and welcomed distractions.

I gratefully acknowledge funding support from the Ryerson International Work Experience Fund (RIWEF), the Ontario Graduate Scholarship (OGS) program, and the Queen Elizabeth II Graduate Scholarship in Science and Technology (QEII-GSST) program.

Finally, and most importantly, I would like to thank my parents, my brothers, my family and friends. The work conducted in this thesis was built on an foundation of loving support and encouragement, and was driven by a thoughtfully nurtured curiosity, for which I am forever grateful. 


\section{Citations to Previously Published Work}

The work presented in Chapter 2 is based off of the following publication:

"Floating and sinking of self-assembled spheres on liquid-liquid interfaces: Rafts versus stacks."

S. G. Jones, N. Abbasi, A. Ahuja, V. Truong, \& S. S. H. Tsai (2015). Physics of Fluids, 27(7), 072102.

In Chapter 3 I present a microfluidic self-assembly system, which is motivated by the study of conformal coating of microparticle clusters. Large portions of the Chapter 3 appear in the following publication:

"Microfluidic magnetic self-assembly of microparticle clusters at an aqueous two-phase liquid-liquid interface" S. G. Jones, N. Abbasi, B. U. Moon, \& S. S. H. Tsai (2015). In preparation.

Outside of the main theme of this thesis, but still motivated by the encapsulation of cells, I present an alternative microfluidic system in Appendix A, which does not involve the coating of microparticles, but instead produces microdroplets. The research presented in Appendix A was led by Dr. Byeong-Ui Moon, and is based on the following publication:

"Microfluidic generation of aqueous two-phase system (ATPS) droplets by controlled pulsating inlet pressures." B. U. Moon, S. G. Jones, D. K. Hwang, \& S. S. H. Tsai (2015). Lab on a Chip, $15(11), 2437-2444$. 


\section{Dedication}

To my little love, Ella. 


\section{Table of Contents}

Author's Declaration . . . . . . . . . . . . . . . . . . . . . ii

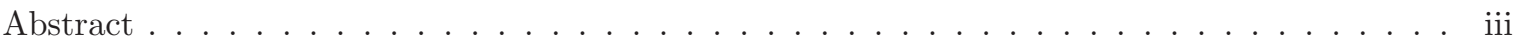

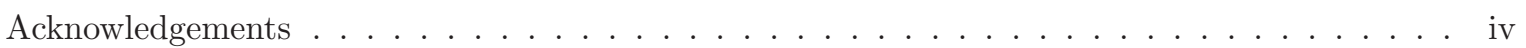

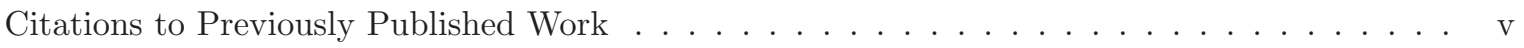

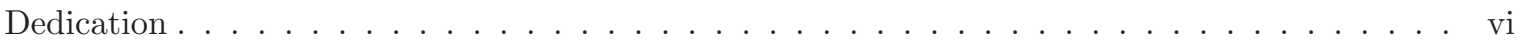

Table of Contents . . . . . . . . . . . . . . . . . . . . vii

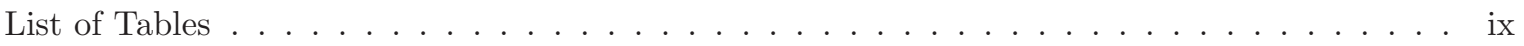

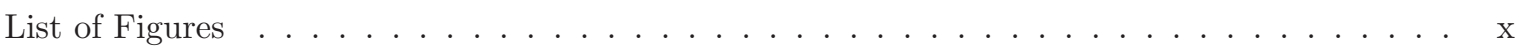

List of Appendices . . . . . . . . . . . . . . . . . . . . xi

List of Symbols . . . . . . . . . . . . . . . . . . . . . . . xii

1 Concepts and Motivation $\quad 1$

1.1 Self-Assembly . . . . . . . . . . . . . . . . . . . . . . . 1

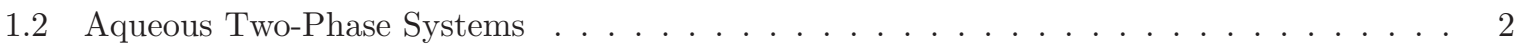

1.3 Cell Transplantation . . . . . . . . . . . . . . . . . . . . . . . . 5

2 Self-Assembled Sphere Clusters $\quad 9$

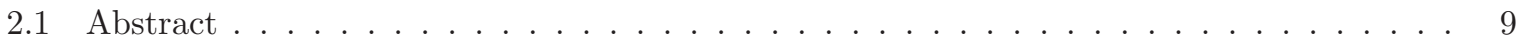

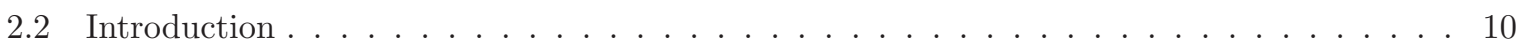

2.3 Experimental Methods . . . . . . . . . . . . . . . . . . . . . . . 10

2.4 Results and Discussion . . . . . . . . . . . . . . . . . . . . . . 12

2.4.1 Assembling interfacial particle rafts and particle stacks . . . . . . . . . . . . 12

2.4.2 Collapse and sinking at above a critical number of spheres . . . . . . . . . . . . 13

2.4.3 Different scaling laws for the critical size of particle rafts and stacks . . . . . . 16

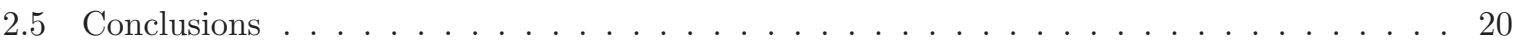

3 Magnetic Microparticle Clusters 23

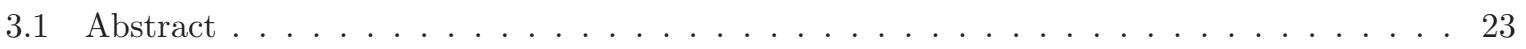

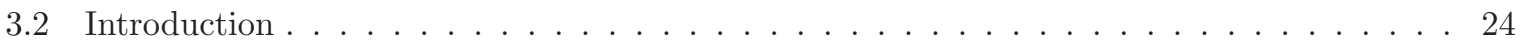

3.3 Experimental Methods . . . . . . . . . . . . . . . . . . . . . . . . 25

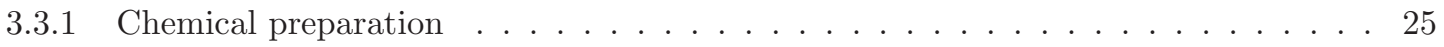




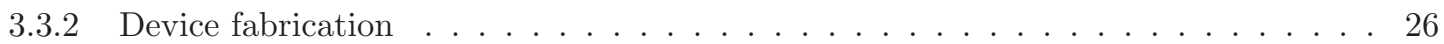

3.3 .3 Experimental setup . . . . . . . . . . . . . . . . . . . 27

3.4 Results and Discussion . . . . . . . . . . . . . . . . . . 30

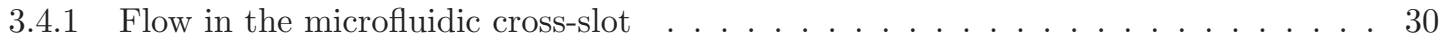

3.4 .2 Single particles at the liquid-liquid interface . . . . . . . . . . . . . 30

3.4 .3 Self-assembly at the liquid-liquid interface . . . . . . . . . . . . . . . 33

3.4.4 Different types of self-assembled clusters . . . . . . . . . . . . . . . 34

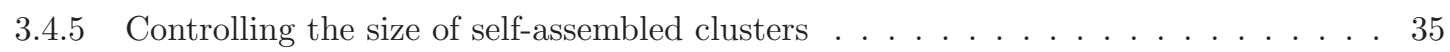

3.4 .6 Coating microparticle clusters . . . . . . . . . . . . . . . . . 40

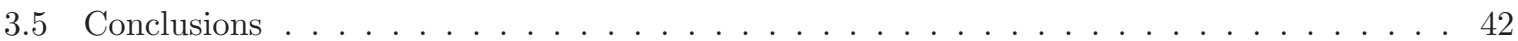

4 Concluding Remarks $\quad 45$

$\begin{array}{ll}\text { Bibliography } & 65\end{array}$ 


\section{List of Tables}

3.1 Composition of the eight dextran-polyethylene glycol ATPS $\ldots \ldots \ldots \ldots$ 


\section{List of Figures}

1.1 Binodal curve for system of two incompatible polymers. . . . . . . . . . . . . . . . 4

1.2 Schematic diagram of a microencapsulated cell cluster. . . . . . . . . . . . . . . 6

2.1 Schematic diagram of sphere cluster deposition geometry. . . . . . . . . . . . . 11

2.2 Experimental images of sphere cluster deposition geometry. . . . . . . . . . . . . 12

2.3 Plot of the critical sphere number $N$ versus individual sphere radius $a$ and interfacial tension $\gamma$. Experimental images of collapsing rafts and stacks. . . . . . . . . . . . 15

2.4 Log-log plots of critical sphere number $N$ versus Bond number Bo. . . . . . . . . . . 18

3.1 Schematic diagram and experimental images of microfluidic chip. . . . . . . . . . . . 28

3.2 Experimental images of a single microparticle passing through the liquid-liquid interface. . 32

3.3 Experimental images of the cross-flow chamber during the magnetic cluster self-assembly. 33

3.4 Experimental images of microparticle clusters passing through the liquid-liquid interface. . 35

3.5 Percentage of microparticle self-assembly type versus magnet distance $\ell_{m} \ldots \ldots \ldots$

3.6 Plot of the cluster particle number $N$ versus magnet position $\ell_{m}$ and interfacial tension

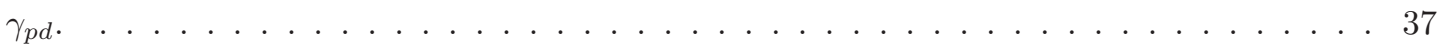

3.7 Log-log plot of the cluster particle number $N$ versus the dimensionless term $C a \cdot \mathrm{Mn}^{-1}$. . 40

3.8 Regime map for single particles, doublets, and triplets. . . . . . . . . . . . . . . 41

A.1 Schematic diagram of ATPS droplet formation in a flow-focusing geometry. . . . . . . . . 52

A.2 Phase diagram of ATPS interfacial dynamics. . . . . . . . . . . . . 54

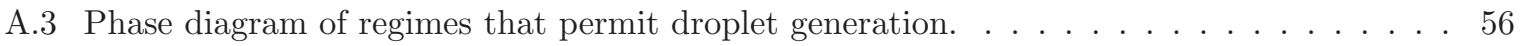

A.4 Size and shape of droplets in the downstream microchannel. . . . . . . . . . . . . . 57

A.5 Plots of measured DEX droplet radius $a$ versus the 'on' and 'off' times of the DEX phase

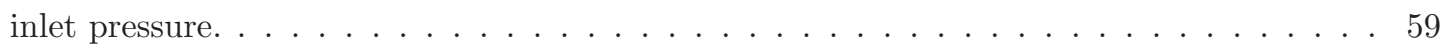

A.6 Log-log plot of the ATPS droplet dimensionless radius $a / w$, versus the dimensionless

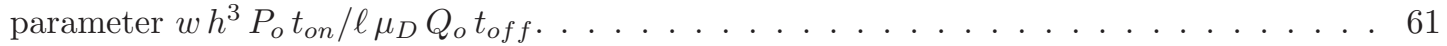

A.7 Experimental image of multiplexed ATPS droplet formation. . . . . . . . . . . . . . 62 


\title{
List of Appendices
}

\author{
Appendix A
}

$\begin{array}{ll}\text { A ATPS Droplet Generation } & 47\end{array}$

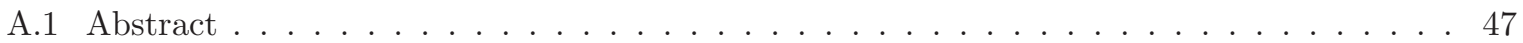

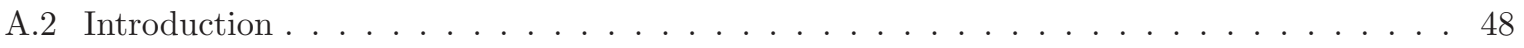

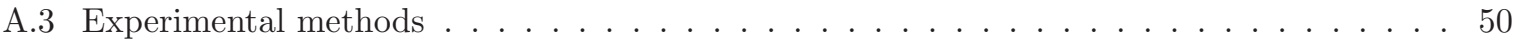

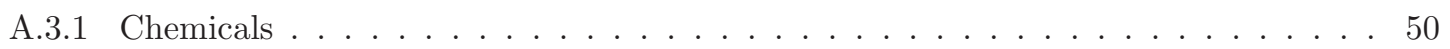

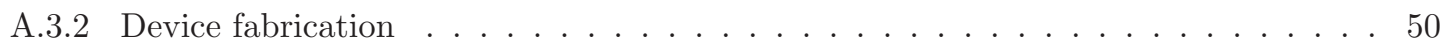

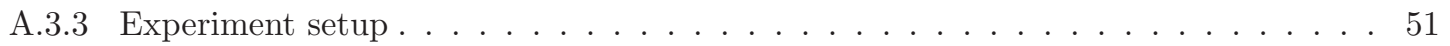

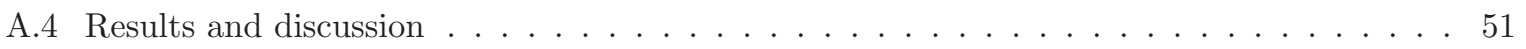

A.4.1 Making ATPS droplets . . . . . . . . . . . . . . . . . . 51

A.4.2 Different regimes of ATPS interfacial dynamics . . . . . . . . . . . . . 53

A.4.3 Elongated ATPS droplet shapes . . . . . . . . . . . . . . . 56

A.4.4 Controlling the size ATPS droplets . . . . . . . . . . . . . . 58

A.4.5 Scaling-up to simultaneous double droplet formation . . . . . . . . . . . . . 61

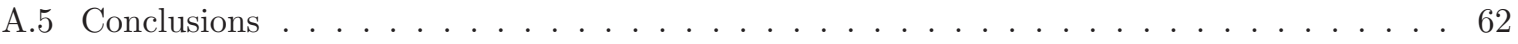




\section{List of Symbols}

\begin{tabular}{|c|c|c|}
\hline Symbol & Units & Description \\
\hline$\alpha$ & - & Dimensionless prefactor \\
\hline$\beta$ & - & Dimensionless prefactor \\
\hline$\gamma, \gamma_{p d}$ & $\mathrm{~N} \cdot \mathrm{m}^{-1}$ & Liquid-liquid interfacial tension \\
\hline$\varepsilon$ & - & Aspect ratio \\
\hline$\eta_{d}, \eta_{o}, \eta_{p}, \eta_{w}$ & $\mathrm{~Pa} \cdot \mathrm{s}$ & Viscosity \\
\hline$\kappa, \kappa^{\prime}$ & - & Dimensionless prefactor \\
\hline$\nu$ & - & Dimensionless flow rate and viscosity ratio \\
\hline$\mu_{d}, \mu_{p}$ & $\mathrm{~Pa} \cdot \mathrm{s}$ & Viscosity \\
\hline$\mu_{o}$ & $\mathrm{~m} \cdot \mathrm{kg} \cdot \mathrm{s}^{-2} \cdot \mathrm{A}^{-2}$ & Permeability of free space \\
\hline$\rho_{o}, \rho_{w}$ & $\mathrm{~kg} \cdot \mathrm{m}^{-3}$ & Liquid density \\
\hline$\rho_{s}$ & $\mathrm{~kg} \cdot \mathrm{m}^{-3}$ & Solid density \\
\hline$\phi$ & - & Angle of inclination of the interface at the inflection line \\
\hline$\chi$ & - & Magnetic susceptibility \\
\hline$\psi$ & - & Angular position of the inflection line relative cluster center \\
\hline$a$ & $\mathrm{~m}$ & Radius \\
\hline Bo & - & Bond number \\
\hline$B o_{m}$ & - & Magnetic Bond number \\
\hline $\mathrm{Ca}$ & - & Capillary number \\
\hline$D$ & - & Density ratio \\
\hline$D_{\max }$ & - & Threshold density ratio \\
\hline $\boldsymbol{F}_{d}$ & $\mathrm{~N}$ & Viscous drag \\
\hline $\boldsymbol{F}_{m}$ & $\mathrm{~N}$ & Magnetic force \\
\hline$g$ & $\mathrm{~m} \cdot \mathrm{s}^{-2}$ & Acceleration due to gravity \\
\hline$h$ & $\mathrm{~m}$ & Height of inflection line \\
\hline$h$ & $\mathrm{~m}$ & Height of microchannel \\
\hline$H$ & - & Aspect ratio \\
\hline $\boldsymbol{H}$ & $\mathrm{A} \cdot \mathrm{m}^{-1}$ & Magnetic field \\
\hline$\hat{H}$ & - & Dimensionless magnetic field \\
\hline
\end{tabular}




$\begin{array}{lll}\ell & \mathrm{m} & \text { Microchannel length } \\ \ell_{c} & \mathrm{~m} & \text { Capillary length } \\ \ell_{m} & \mathrm{~m} & \text { Length from magnet face to interface } \\ \ell_{r}, \ell_{s} & \mathrm{~m} & \text { Characteristic length } \\ \ell_{w} & \mathrm{~m} & \text { Microchannel width } \\ M & \mathrm{~A} \cdot \mathrm{m}^{-1} & \text { Magnetization of permanent magnet } \\ M n & - & \text { Mason number } \\ N & - & \text { Critical number of spheres } \\ P, P_{o} & \mathrm{~Pa} & \text { Pressure } \\ Q_{d}, Q_{o}, Q_{p} & \mathrm{~L} \cdot \mathrm{s}^{-1} & \text { Flow rate } \\ t, t_{\gamma}, t_{e q}, t_{o f f}, t_{o n} & \mathrm{~s} & \text { Time } \\ t & - & \text { Aspect ratio } \\ U & \mathrm{~m} \cdot \mathrm{s}^{-1} & \text { Average flow speed } \\ u & \mathrm{~m} \cdot \mathrm{s}^{-1} & \text { Flow speed } \\ v & \mathrm{~m} \cdot \mathrm{s}^{-1} & \text { Characteristic velocity } \\ \hat{v} & - & \text { Dimensionless velocity } \\ w, w_{c} & \mathrm{~m} & \text { Microchannel width } \\ x, y & \mathrm{~m} & \text { Unit direction } \\ \hat{y} & - & \text { Dimensionless length }\end{array}$




\section{Chapter 1}

\section{Concepts and Motivation}

\subsection{Self-Assembly}

The self-assembly of objects is a fascinating phenomenon, where parts spontaneously organize into more complex shapes via an energy-minimization process. The term self-assembly is very broad, and is used to describe a wide variety of processes, which can be driven by a range of different forces. For example, deoxyribonucleic acid molecules (DNA) can be designed to form specific crystal structures, where the molecules self-assemble due to intermolecular interactions [1]. Charged polyelectrolytes can self-assemble on surfaces to form uniform coating layers [2]. Amphiphilic molecules, such as lipids, which have both a hydrophilic end and a hydrophobic end, can spontaneously assemble into spherical micelles [3, 4]. These kinds of molecular scale self-assembly systems have huge potential in nanotechnology applications, where traditional manufacturing processes break down $[1,5]$. A review article by Whitesides and Grzybowsk attempts to capture the diversity of self-assembly systems [6].

In this thesis, I am interested in the self-assembly of monodisperse colloidal particles on liquid-liquid interfaces. The self-assembly of particles on a fluid-liquid interface has been coined "the cheerios effect" [7], in reference to how cereal on the surface of milk tends to aggregate into a cluster in the center of 
the bowl, and at the edges of the bowl. This specific kind of self-assembly is driven by a combination of gravity and capillarity. In Chapter 2 I investigate the self-assembly of sphere clusters on a liquid-liquid interface due to this type of self-assembly. In particular, I investigate how large the self-assembled sphere clusters can grow before they sink through the liquid-liquid interface, and the effect of the deposition geometry on the critical number of spheres that causes sinking.

Moreover, I am interested in replicating many of the qualities of the sphere cluster self-assembly system, such as the precise size control, in a microfluidic flow. However, in most micron length-scale systems the effect of gravity is negligible, due to the increasing surface-to-volume ratio at smaller length scales. As a result, gravity is not an ideal body force to drive particle self-assembly of microparticles in a microfluidic device. Instead, I apply a magnetic field gradient to drive the self-assembly of paramagnetic microparticles, a so-called magnetocapillary self-assembly system $[8,9]$.

In Chapter 3, I present the microfluidic system which self-assembles paramagnetic microparticles into clusters on a liquid-liquid interface. Similar to the aforementioned sphere clusters, I am interested in controlling the number of particles within a single cluster, and the entrainment of fluid by the particle cluster. Since the magnitude of the body force provided by the magnetic field gradient is still relatively weak, the microfluidic self-assembly system will also require using a liquid-liquid interface with an ultralow interfacial tension $[9,10]$.

\subsection{Aqueous Two-Phase Systems}

Many multiphase microfluidic systems utilize a combination of oil and water to produce a liquid-liquid interface. The interfacial tension of the oil-water interface can be reduced to an ultra-low interfacial tension with the use of surfactants. However, the organic oil phase is typically toxic to cells, and in biological applications the oil phase must be carefully removed in a post-processing step. As a result, there has been a renewed interest in aqueous two-phase systems (ATPS), due to the natural ultra-low 
interfacial tension of the aqueous liquid-liquid interface, and the biocompatibility of the immiscible liquid phases.

ATPS consists of two incompatible polymers dissolved in water, which naturally phase separate above a critical dissolved polymer concentration. There are numerous polymer combinations that exhibit this phase separation behavior, but one of the most commonly studied solutions is a mixture of polyethylene glycol (PEG) and dextran (DEX) [11, 12]. ATPS have been used historically for batch separation processes which involve the collection of fragile biomolecules or cell organelles. The disintegration of cells produces a complex solution with a variety of particle sizes. A specific ATPS can be purposely selected such that the target biomolecule preferentially partitions to one of the two aqueous phases, or collects at the liquid-liquid interface between the two aqueous phases. Not only does this method enable the separation of specific biomolecules, but the process is also extremely gentle in comparison to the alternative centrifugation techniques [11].

The phase separation behavior of a specific ATPS is characterized by a binodal curve. Figure 1.1 shows a generic binodal curve for two hypothetical polymers. A solution prepared with a total polymer concentration which appears above the binodal curve will phase separate into two distinct phases, whereas a polymer composition below the curve will remain as a single homogeneous solution. Immediately after the solution is prepared, it begins to slowly separate into the two immiscible fluid phases. The composition of the two equilibrated phases is indicated by the intersection of the tie-line and the binodal curve (see Fig. 1.1). For example, the composition of an ATPS with a total polymer concentration indicated by point A, would phase separate into two phases with the compositions B and C. For this hypothetical ATPS, the equilibrated phase B consists of mostly polymer Q, and would be refereed to as the polymer Q-rich phase, or simply the polymer Q phase (for a PEG-DEX ATPS, the equilibrated solutions are referred to as the PEG phase and the DEX phase). A solution with a total polymer composition which lies on a different location of the BC tie line will still produce phases with compositions $\mathrm{B}$ and $\mathrm{C}$, but with a different volume ratio [11]. 


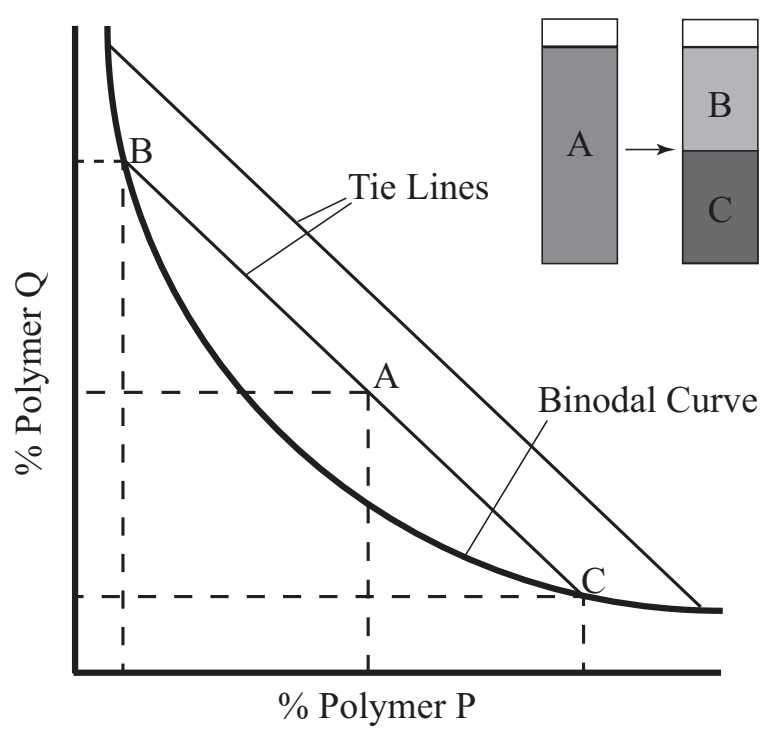

Figure 1.1: Binodal curve for a system of two incompatible polymers. An aqueous solution prepared with a weight fraction of polymers $\mathrm{P}$ and $\mathrm{Q}$ indicated by point $\mathrm{A}$ will phase separate into two immiscible phases with compositions B and C.

The shape of the binodal curve is sensitive to a variety of controllable parameters, such as the polymer molecular weight, solution temperature, and the relative hydrophobicity of the two polymers. An excellent reference text by Albertsson discusses the phase separation behavior of ATPS, and how to exploit these characteristics for the selective partitioning of biomolecules [11].

The liquid-liquid interface between equilibrated ATPS phases typically has an ultra-low interfacial tension, which can be tuned by adjusting the dissolved polymer concentration. Each binodal curve has a family of parallel tie lines (only two shown in Fig. 1.1), which tend to get longer with an increasing total dissolve polymer concentration. The interfacial tension of the ATPS liquid-liquid interface has been shown to be proportional to the logarithm of the tie-line length $[13,14]$. As a result, ATPS are particularly well suited for the systematic study of microparticle self-assembly and coating, which has been demonstrated previously on oil-water interfaces with ultra-low interfacial tension $[9,15]$. 


\subsection{Applications in Cell Transplantation and Immunoisolation}

One of the biomedical applications of the conformal coating of microparticle clusters is in cellular transplantation. In this application, the conformal coating layer, which forms around the particle cluster as it passes through the liquid-liquid interface, is polymerized and used as a semipermeable membrane. Similarly, cells which are magnetized by functionalized magnetic particles could be forced through a liquid-liquid interface, where the subsequently formed conformal coating layer is polymerized downstream. This semipermeable polymer layer functionally isolates the coated cells from their local external environment, which has the potential to serve as a local immunosuppressant.

Cellular transplantation is a proposed treatment for a variety of complex diseases. For example, Diabetes mellitus type 1 is an autoimmune disorder which destroys the insulin producing beta cells of the pancreas. As a result of the lack of insulin, patients with type 1 diabetes are not able to properly regulate their blood glucose levels. This disorder is often managed with daily insulin injections, but requires periodic measurements of the blood glucose levels and self-injection of insulin. An alternative treatment consists of transplanting new pancreatic islets into the patient, which are able to excrete insulin in direct response to increased blood glucose levels. However, implanting donor cells into a patient inevitably causes a reaction from the host's immune system, which is traditionally suppressed with the use of systemic immunosuppressant drugs [16]. Though effective, these systemic drugs have a wide range of side-effects which negatively impact the patient's quality of life.

Cellular encapsulation is an alternative immunoisolation technique, where the transplanted cells are protected locally by a semipermeable polymeric membrane, which isolates the cells from the immune system (see schematic diagram in Fig. 1.2). There are a variety of devices that are under development that serve this purpose [17], but here I will focus specifically on polymeric microencapsulation.

Microencapsulation is the process of encapsulating a cell or a cell cluster in a biocompatible polymeric coating layer, such as alginate or polyethylene glycol. The coating layer porosity is tuned to allow the 


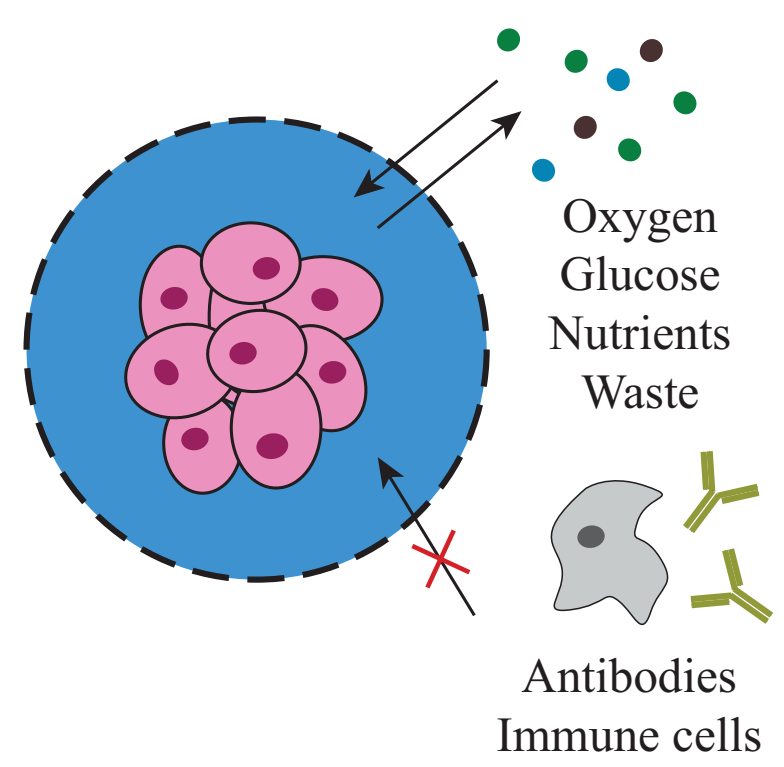

Figure 1.2: Schematic diagram of a microenapsulated cell cluster. The semi-permeable polymeric coating allows the diffusion of essential metabolic molecules, such as oxygen and glucose, but does not allow the diffusion of the larger macromolecules of the immune system. As a result, the transplanted cell cluster is able to maintain cellular homeostasis in the host patient, without the use of immunosuppressant drugs. 
diffusion of oxygen, glucose, and other essential metabolic molecules through the coating membrane, while preventing the passage of the large macromolecules of the immune system. While there are several interesting problems that need to be solved before this type of cell encapsulation can be used clinically, the application of a coating layer itself is a very traditional fluid mechanics problem.

Today, most encapsulation techniques produce a relatively thick coating layer, which inhibits the diffusion of metabolic molecules across the membrane to the cell cluster. For example, a suspension of cells can be formed into droplets in a microfluidic flow-focusing geometry, where each microdroplet produced contains a single cell. This method of cellular encapsulation produces a coating with a thickness of $\mathrm{O}(10-100) \mu \mathrm{m}[18,19,20]$. In comparison, coating particles via magnetic forcing has been shown to produce a conformal coating layer which is $\mathrm{O}(1) \mu \mathrm{m}$ in thickness [9]. In Chapter 3, I present a microfluidic system which self-assembles magnetic microparticles on a liquid-liquid interface. I then investigate the parameters that control the cluster passage through the interface, and the nature of the coating layer formed upon interfacial passage.

In addition, in Appendix A I present a microfluidic system for producing monodisperse water-in-water droplets from equilibrated ATPS, which could also be used for the encapsulation of cells. Encapsulation of cells within the ATPS droplets does not require an oil phase, and does not rely on magnetic particles to coat the cells, but it produces a coating thickness that is comparable to existing cell encapsulation techniques. However, the system is very simple to set up and use. This work was led by Dr. ByeongUi Moon, where I was responsible for producing the ATPS used during the experiments and provided knowledge of the characteristics and applications of ATPS solutions. 



\section{Chapter 2}

\section{Floating and sinking of self-assembled spheres on liquid-liquid interfaces: rafts versus stacks}

\subsection{Abstract}

The floating and sinking of objects on fluid-fluid interfaces occurs in nature, and has many important implications in technology. Here, I study the stability of floating self-assembled spheres on an oil-water interface, and how the sphere deposition geometry affects the size limits of the assemblies before they collapse and sink through the interface. Specifically, I compare the critical size of particle rafts to particle stacks. I show that, on liquid-liquid interfaces, monolayer rafts and stacked spheres exhibit different scaling of the critical number of spheres to the Bond number - the dimensionless ratio of buoyancy to interfacial tension effects. My results indicate that particle stacks will sink with a lower threshold number of particles than particle rafts. This finding may have important implications to engineering applications where interfacial assemblies are not monolayers. 


\subsection{Introduction}

The floating and sinking of objects on fluid-fluid interfaces is of practical importance in many applications [21]. In nature, water striders and other insects depend on the support of surface tension and buoyancy to stand above the free interface of water $[22,23]$. Fire ants link their bodies together to improve their collective water repellency, so that they can stay afloat and avoid drowning [24, 25]. In technology, water-walking robots exploit the dependency of the force from surface tension on the robot's surface area, to increase their load carrying capacity [26, 27, 28]. Gravity-induced destabilization of granular rafts and colloid monolayers on fluid-fluid interfaces may be used to encapsulate oils [29] and create Pickering emulsions [30, 31], respectively.

In this chapter, I consider the self-assembly and destabilization of monodisperse particles at a liquidliquid interface, and attempt to address the question of how large such assemblies can get before they sink. While related problems have been studied previously in two $[32,33,34]$ and three $[8,29]$ dimensions, those systems only consider monolayer particle rafts. Here, I ask the additional question of what happens when the particle deposition geometry changes; namely, how results change if particles are loaded on top of each other on the liquid-liquid interface (as opposed to particles placed adjacent to each other in forming monolayer rafts).

I first report my experimental findings, then I compare my results to previous calculations of the threshold particle density ratio that identifies collapsible and arbitrarily large rafts and stacks. Finally, for collapsible systems, I develop simple scaling models based on the dimensionless Bond number, for the critical number of spheres that trigger interfacial sinking. I find distinctively different power-laws for particle rafts and stacks, which agree with my experimental observations. My work also reveals that particle stacks collapse with a lower threshold number of spheres, when compared to particle rafts.

\subsection{Experimental Methods}

In experiments, I fill a glass container that has equal side-lengths, $15 \times 15 \times 15 \mathrm{~cm}$, with $2 \mathrm{~L}$ of deionized (DI) water and $1 \mathrm{~L}$ of olive oil (Marque Gallo Brand, Portugal). The DI water and olive oil have densities $\rho_{w}=1,000$ and $\rho_{o}=911 \mathrm{~kg} / \mathrm{m}^{3}$, respectively, and viscosities $\eta_{w}=1$ and $\eta_{o}=84 \mathrm{mPa}$, respectively. 
(a)

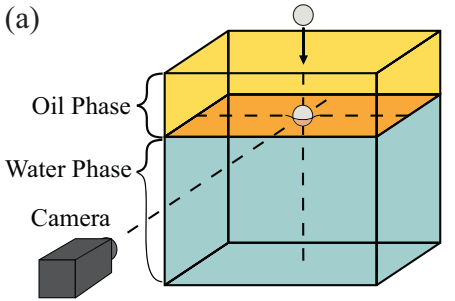

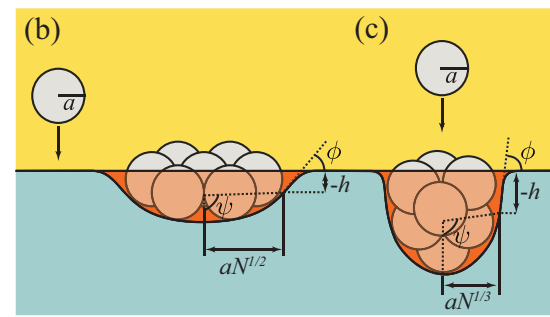

Figure 2.1: (a) Nylon spheres are deposited, one-at-a-time, from above the glass container. Each sphere descends through the oil phase and settles at the oil-water interface. The spheres attract via a combination of interfacial capillary and buoyancy effects, to form a cluster. The cluster is allowed to completely settle at the liquid-liquid interface before additional spheres are added. (b) A monolayer raft is formed by placing new spheres away from the spheres that are already on the oil-water interface. (c) When spheres are stacked on top of each other, they form an assembly that has an approximate spheroidal shape.

Before I deposit spheres on the oil-water interface, the oil-water system is allowed to settle for $\sim 2$ hours so that the interface becomes completely stable.

Nylon spheres (Precision Plastic Ball Co., Franklin Park, IL, USA), with density $\rho_{s}=1,150 \mathrm{~kg} / \mathrm{m}^{3}$, and radii $a=1.2$ to $3.2 \mathrm{~mm}$, are deposited from above the oil phase in the container one-at-a-time. The spheres descend through the oil phase and settle on the liquid-liquid interface (Fig. 2.1 (a)). I wait approximately 20 seconds until the self-assembled spheres have reached a stable state before adding more spheres to the assembly. Prior to each experiment the spheres are washed in an isopropyl-based cleaning agent, and air dried.

I modify the interfacial tension of the oil-water interface by adding the surfactant sodium dodecyl sulfate (SDS, Sigma-Aldrich, St. Louis, MO, USA) to the DI water, and measure the interfacial tension using the pendant drop method [35]. I find the oil-water interfacial tensions, $\gamma=11.7$ (4 mM SDS), 14.5 (2 mM SDS), 18.5 (1 mM SDS), and $24.9(0 \mathrm{mM} \mathrm{SDS}) \mathrm{mN} / \mathrm{m}$.

I use a digital SLR camera (Nikon D90, Tokyo, Japan) with a marco lens (Nikkor 85 mm f/3.5G, Tokyo, Japan) to image the spheres' interfacial self-assembly and eventual sinking. The camera is set on a tripod and focused at the center of the oil-water interface (see Fig. 2.1 (a)). I apply LED back-lighting (Edmund Optics, Barrington, NJ, USA) to improve the image contrast. 


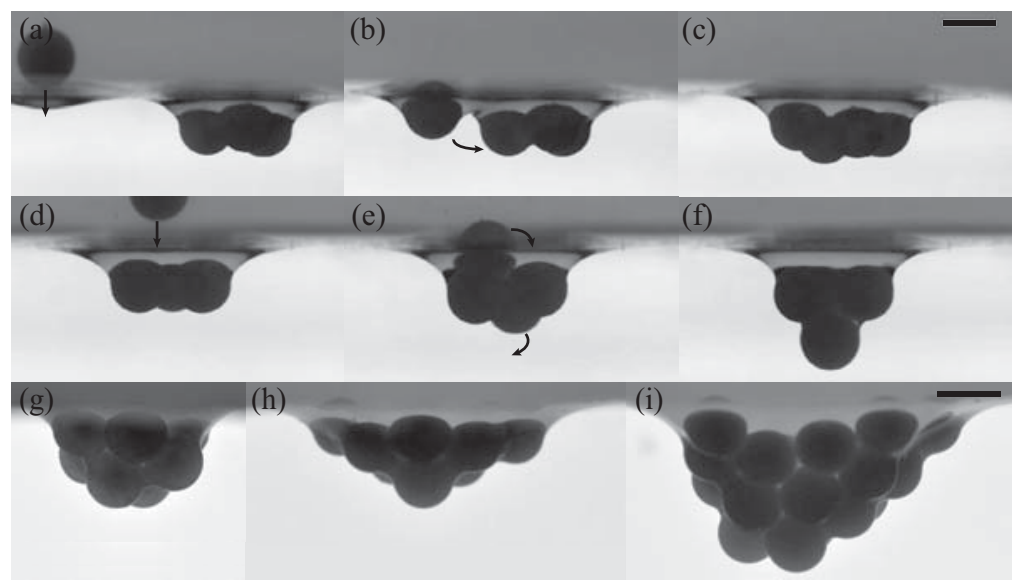

Figure 2.2: Spheres of radius $a=2.8 \mathrm{~mm}$, assemble on an oil-water interface with interfacial tension $\gamma=$ $24.9 \mathrm{mN} / \mathrm{m}$. In making interfacial rafts, (a) a sedimenting sphere descends to the liquid-liquid interface away from other spheres, (b) the sphere gets pulled to the particle raft by a combination of interfacial deformation and gravity, and (c) the sphere self-assembles with the other spheres of the existing raft. When I stack the spheres on the liquid-liquid interface, (d) a sphere will descend on top of the spheres that are already at the interface, (e) often resulting in an instability and subsequent cluster rotation, and (f) finally settle into a quasi-equilibrium state at the interface. Large clusters of 10 spheres, each with radius $a=2.2 \mathrm{~mm}$, assemble in equilibrium by the (g) raft and (h) stack methods on the oil-water interface. Here, the shape of the equilibrated liquid-liquid interface is much more deformed in supporting a particle stack than a raft of the same number of spheres. The stacked cluster collapses at critical sphere number $N=11$, and (i) the sphere raft supports up to 23 spheres (before collapsing at $N=24$ spheres). Scale bar indicates $5 \mathrm{~mm}$.

\subsection{Results and Discussion}

\subsubsection{Assembling interfacial particle rafts and particle stacks}

The two different methods that I use to deposit the spheres result in the formation of sphere rafts (Fig. 2.1 (b)) and sphere stacks (Fig. 2.1 (c)). In forming rafts, I place new spheres away from spheres that are already at the interface (Fig. 2.2 (a)). Depositing new spheres on the oil-water interface causes the spheres to self-assemble by a combination of interfacial deformation and gravity, to form a monolayer on the liquid-liquid interface (Figs. 2.2 (b) and (c)). This type of interfacial particle self-assembly has been studied previously [36, 37], and it is termed "the cheerios effect" in reference to how cereals tend to cluster on the free interface of a bowl of milk [7]. 
In Fig. 2.2, I differentiate the stacking configuration by the deposition of new spheres on top of existing spheres at the interface (Fig. 2.2 (d)). Maintaining a new sphere vertically on top of an existing interfacial cluster often results in an instability, where the entire stack will rearrange and rotate until it arrives at a more energetically favorable state (see example Figs. 2.2 (e) and (f)).

Figs. $2.2(\mathrm{~g})$ and $(\mathrm{h})$ show stack and raft clusters, respectively; each cluster has 10 spheres in equilibrium on the liquid-liquid interface. Here the sphere radius $a=2.2 \mathrm{~mm}$, and the liquid-liquid interfacial tension $\gamma=24.9 \mathrm{mN} / \mathrm{m}$. The equilibrated liquid-liquid interface is much more deformed in supporting a particle stack than a raft of the same number of spheres. For stacked sphere clusters, the interface consistently maintains an approximately spheroidal assembly (Fig. $2.2(\mathrm{~g})$ ); whereas liquidliquid interfaces that support monolayer particle rafts take on a shape that is more similar to a curved elastic sheet (Fig. $2.2(\mathrm{~h})$ ). In this particular case, the stack sinks with a critical size $N=11$, while the raft continues to support up to 23 spheres (Fig. 2.2 (i)). When the final sphere $(N=24)$ is added to the raft, the raft collapses and passes through the interface.

\subsubsection{Collapse and sinking at above a critical number of spheres}

Previous calculations [33] had showed that, in a two-dimensional geometry, where the aspect ratio $t=a / \ell_{c}$ accounts for the finite radius $a$ of the particles and the capillary length $\ell_{c}=\left(\gamma /\left(\rho_{w}-\rho_{o}\right) g\right)^{1 / 2}$, the ratio $D=\left(\rho_{s}-\rho_{o}\right) t /\left(\rho_{w}-\rho_{o}\right)$ determines whether a floating raft can grow arbitrarily large without ever sinking (when $D<D_{\max }$ ), or eventually sink at above a critical particle number (when $D>D_{\max }$ ). Here, $g=9.81 \mathrm{~m} / \mathrm{s}^{2}$ is the acceleration due to gravity, and $D_{\max }$ is the threshold density ratio. The ratio $D$ compares the depth of the interfacial deformation caused by the presence of the particles, to the capillary length $\ell_{c}$.

As described in more detail in a review by Vella [21], $D<D_{\max }$ corresponds to the condition where the relative density of the particles is so low that the addition of more particles would simply lower the entire assembly, and the increase in hydrostatic pressure would accommodate the additional particles. When $D>D_{\max }$, the relative density of the particles is sufficiently large that, at a critical number of particles, the aggregate weight from all of the particles lowers the assembly by an amount that is greater than the capillary length $\ell_{c}$, which causes the assembly to sink. 
The inset in Fig. 2.3 (a) shows a plot of the three-dimensional system's threshold density ratio $D_{\max }$ versus the interfacial tension $\gamma \cdot \times$ and + indicates values obtained from experiments of raft and stack configurations, respectively. For each value of interfacial tension $\gamma$, I systematically vary the sphere radius $a$ as I assemble interfacial rafts and stacks. I find that the threshold value $D_{\max } \approx 1$ in all of my three-dimensional raft and stack experiments.

I obtain this result by assuming that I attain an infinite raft or stack when I deplete my supply of (more than 100) spheres, or when the cluster extends to the boundaries of the glass tank, without triggering an instability and sinking. Notably, I observe similar values of the threshold density ratio $D_{\max }$ in both sphere raft and sphere stack experiments. My experimental value $D_{\max } \approx 1$ is similar to the previous calculation $D_{\max }=\sqrt{2}$, which was made for a two-dimensional geometry [33], and my result agrees with Abkarian et al. [29], who found that axisymmetric monolayer particle rafts do not sink when $D<<1$.

In the regime where $D>D_{\max }$, instability is initiated once the floating raft or stack reaches a critical sphere number, $N$. Fig. 2.3 (a) shows a plot of the critical sphere number, $N$, versus individual sphere radius, $a$. Solid symbols show data from raft experiments, and empty symbols reflect results from stacking spheres on the oil-water interface. For each configuration, I also vary the liquid-liquid interfacial tension, $\gamma$, to observe how the interfacial restoring energy affects the critical sphere number $N$. Each data point on the plot is averaged from ten experiments, and error bars represent one standard deviation.

I observe that the critical number of spheres $N$ decreases monotonically with increasing sphere radius $a$, and grows monotonically as the oil-water interfacial tension $\gamma$ is increased. With sphere radius $a$ and interfacial tension $\gamma$ fixed, clusters in the raft configuration tend to grow to a larger sphere number than stacked clusters, before collapsing and sinking through the liquid-liquid interface.

Figs. 2.3 (b) and (c) show representative image sequences of collapsing rafts and stacks, respectively, immediately after the onset of instability. Here the interfacial tension $\gamma=24.9 \mathrm{mN} / \mathrm{m}$, and the sphere radius $a=2.8 \mathrm{~mm}$. The collapse initiates at the location of the critical sphere, and propagates to pull in the surrounding spheres. As the cluster descends through the lower water phase, an interfacial neck forms, becomes thinner, and eventually ruptures, while the cluster entrains a thin coating film of the 

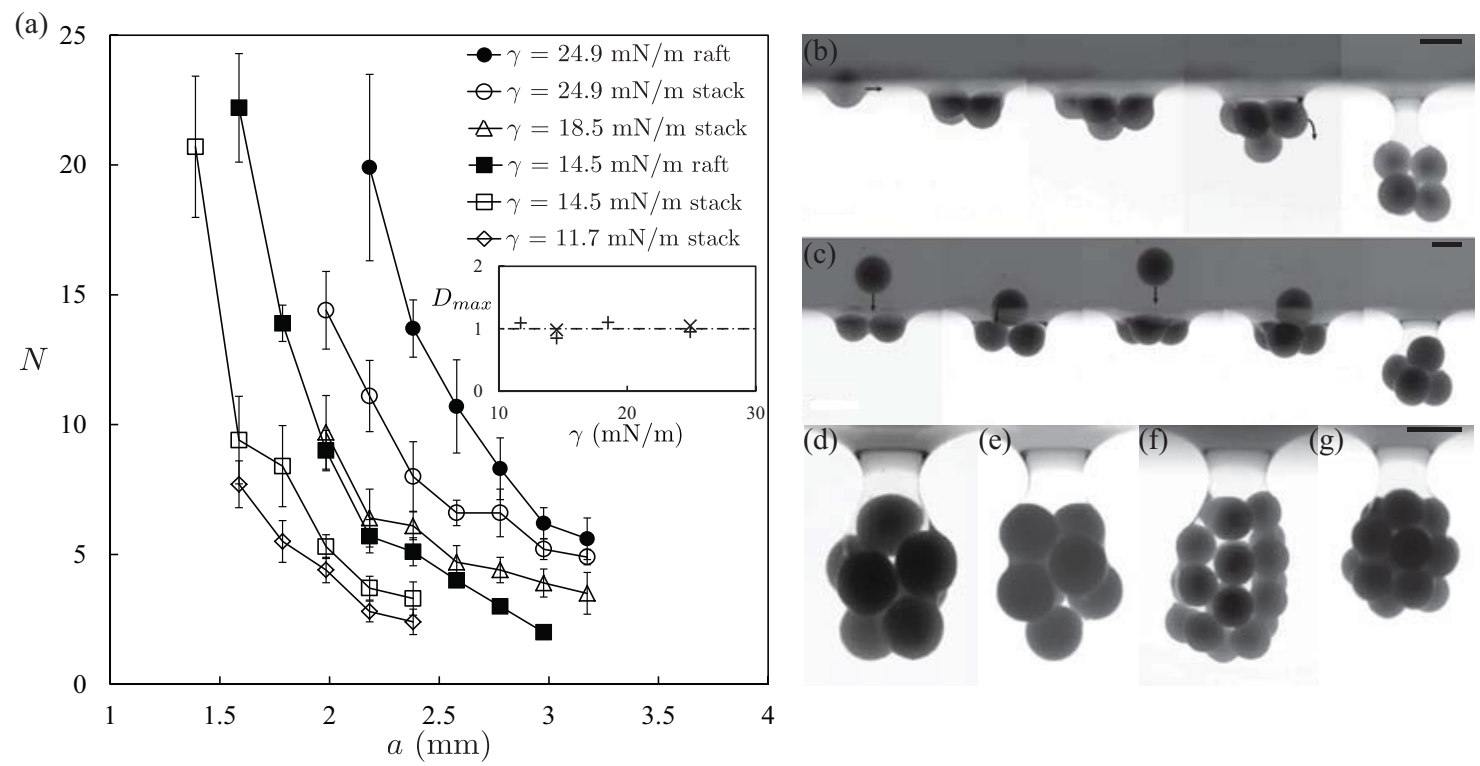

Figure 2.3: Sphere rafts and stacks of different sizes are formed on an oil-water interface. The rafts and stacks eventually collapse and sink through the interface when the cluster size reaches a critical sphere number, $N$. (a) The critical sphere number $N$ varies with changes in the sphere radius $a$, the oil-water interfacial tension $\gamma$, and the deposition configuration (either raft or stack). I find that the cluster size $N$ grows monotonically with decreasing sphere size $a$, and with increasing interfacial tension $\gamma$. I also find that spheres deposited into a monolayer raft configuration sinks through the interface as a larger cluster than spheres stacked into a spheroidal stack. Error bars represent one standard deviation. The inset shows the threshold ratio $D_{\max }$ versus interfacial tension $\gamma \cdot \times$ and + indicates values obtained from experiments of raft and stack configurations, respectively. Image sequences of spheres with radius $a=2.8$ $\mathrm{mm}$, assembling into (b) rafts and (c) stacks on a liquid-liquid interface, with interfacial tension $\gamma=24.9$ $\mathrm{mN} / \mathrm{m}$. At above the threshold density ratio $D_{\max }$, both particle rafts and stacks eventually collapse and sink through the liquid-liquid interface. At low sphere numbers, $(N<10)$ both (d) rafts and (e) stacks form similar spheroidal packing arrangements. At larger sphere numbers the packing geometry can differ more significantly between (f) rafts and (g) stacks, although both retain an approximate spheroidal shape. Scale bars represent $5 \mathrm{~mm}$. 
upper oil phase. I anticipate that the coating film thickness will scale with the cluster passage speed, since more rapid passage results in reduced time for the liquid to drain between the cluster and the liquid-liquid interface. Additionally, both collapsed rafts (Fig. 2.3 (d)) and stacks (Fig. 2.3 (e)) feature the same approximate spheroidal geometry.

Several recent studies have investigated the packing geometry of small hard sphere clusters resulting from energy minimization [38, 39], such as clusters encapsulated in shrinking droplets [39]. I observe that in my experiments collapsed sphere clusters often organize into predicted geometries (for example Figs. 2.3 (d) and (e) form a pentagonal diamond [39]), however in some cases the clusters form packing arrangements that are not energetically optimal (for example in Fig. 2.3 (c) the collapsed cluster with $N=4$ spheres forms a tetrahedron, but in Fig. 2.3 (b) it does not). I attribute this variation in the cluster geometry to the asymmetrical confinement of the clusters by the liquid-liquid interface during my experiments. For collapsed clusters with $N>10$ spheres, the packing geometry of the spheres can vary considerably, although collapsed clusters still maintain an approximate spheroidal shape (e.g. Figs. $2.3(\mathrm{f})$ and $(\mathrm{g}))$.

\subsubsection{Different scaling laws for the critical size of particle rafts and stacks}

To understand at what critical size a particle raft begins to sink, I adapt the generalized equations of Archimedes' principle by Keller [40], and mathematical arguments made by Vella et al. [32] to my multisphere geometry (Fig. 2.1 (b) and (c)). I measure the particle Reynolds number Re, the dimensionless ratio of inertia to viscous effects, and the particle Weber number $W e$, the dimensionless ratio of inertia to interfacial tension, and find that $R e$ and $W e$ are both $\mathrm{O}\left(10^{-1}\right)$. For this reason I neglect the effect of inertia in my analysis. Keller has shown that the vertical component of the interfacial tension force acting on a body floating at a fluid-fluid interface is equal to the weight of the fluid displaced my the meniscus around the body [40]. Vella utilizes this principle and develops an expression for the balance of the weight of a floating sphere at a fluid-fluid interface, to the restoring forces of interfacial tension and buoyancy [32]. I adapt this expression for my multi-sphere raft geometry, such that, 


$$
\frac{4}{3} \pi N g a^{3}\left(\rho_{s}-\rho_{o}\right)=2 \pi \ell_{r} \gamma \sin \psi \sin \phi+\pi N g a^{3}\left(\rho_{w}-\rho_{o}\right)\left(-\frac{h}{\ell_{r}} \sin ^{2} \psi+\frac{2}{3}-\cos \psi+\frac{1}{3} \cos ^{3} \psi\right) .
$$

Here, the particle raft characteristic length $\ell_{r}$, the height of the interfacial inflection line above the undeformed interface $h$, the angle of inclination of the interface at the inflection line $\phi$, and the angular position of the inflection line relative to the center of the particle raft $\psi$ (see Fig. 2.1). The height $h$ and the angles $\phi$ and $\psi$ are used to define the shape of the deformed liquid-liquid interface due to the presence of a floating sphere.

The left side of (2.1) balances gravity acting on the entire raft to the restoring forces on the right: force from interfacial tension (first term) and buoyancy force (second term). I note that the force from interfacial tension scales with the circumference of the particle raft, and the buoyancy and gravity terms are both based on the raft volume. I have added the critical sphere number $N$ to the gravity and buoyancy terms to account for multiple spheres at the liquid-liquid interface, and I use $\ell_{r}$ to calculate the circumference of the raft. I also assume that the spheres only wet the oil phase, so that a three-phase contact line is not present. This assumption is supported by my observations that, upon destabilization and sinking, the nylon spheres become completely coated with a thin film of oil.

I approximate the raft characteristic length $\ell_{r}$ using the raft coverage area such that $\ell_{r}^{2} \approx N a^{2}$. As a result, the raft characteristic length $\ell_{r} \approx a N^{1 / 2}$ (Fig. 2.1 (b)). Then I define the Bond number $B o=\left(\rho_{s}-\rho_{o}\right) g a^{2} / \gamma$, the aspect ratio $H=h / \ell_{r}$, and reorganize (2.1) to obtain,

$$
N=\alpha B o^{-2}+\beta B o^{-1}
$$

where the $O(1)$ pre-factors,

$$
\alpha=\frac{36 \sin ^{2} \psi \sin ^{2} \phi}{\left(2-4 D / t-3 \cos \psi+\cos ^{3} \psi\right)^{2}} \quad \text { and } \quad \beta=\frac{9 H^{2} \sin ^{4} \psi}{\left(2-4 D / t-3 \cos \psi+\cos \psi^{3}\right)^{2}} .
$$



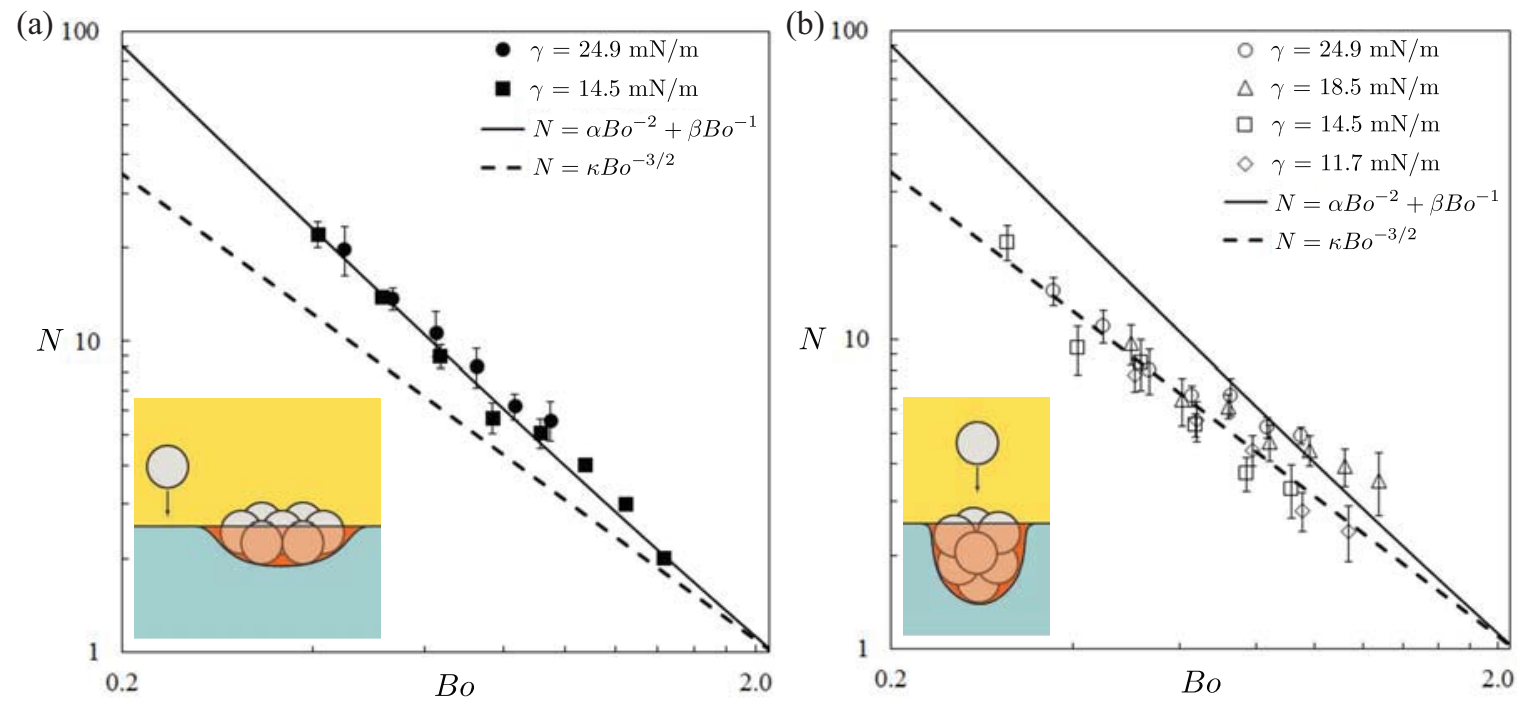

Figure 2.4: Log-log plots of critical sphere number $N$ versus Bond number $B o$, giving two different power laws for sphere raft and stack configurations. (a) The solid line shows that clusters assembled in a raft configuration sink through the oil-water interface at a critical sphere number $N=\alpha B o^{-2}+\beta B o^{-1}$. (b) The dashed line shows that, when I stack spheres on the oil-water interface, the resulting critical sphere number $N=\kappa B o^{-3 / 2}$.

These simplifications result in the scaling relationships, $N \propto B o^{-2}$ when Bond number $B o$ is small (i.e. restoring force dominated by interfacial tension), and $N \propto B o^{-1}$ when Bond number $B o$ is large (i.e. buoyancy effects important).

Fig. 2.4 (a) shows a log-log plot of the critical sphere number $N$ versus the Bond number $B o$ in my experiments based on a raft geometry (see inset schematic). By fitting once with $\alpha \approx 3.5$ and $\beta \approx 0.5$ in (2.2), I find an excellent agreement between the experiments and my model. I note that, over the range of Bond numbers $B o$ in my experiments, interfacial tension plays a much more significant restoration role than buoyancy effects: the model resembles $N \propto B o^{-2}$ more closely than $N \propto B o^{-1}$ in the scope of my observations. The scaling $N \propto B \mathrm{~B}^{-2}$ also appears in other related studies of interfacial raft formation where buoyancy effects is neglected $[8,30]$, but my more generalized model $(2.2)$ will be applicable in higher ranges of the Bond number Bo, where buoyancy plays a more significant role than interfacial tension. 
In the sphere stacking configuration, I use the stack volume $\ell_{s}^{3} \approx N a^{3}$ to extract the stack characteristic length $\ell_{s} \approx a N^{1 / 3}$ (see Fig. $2.1(\mathrm{c})$ ). Replacing $\ell_{r}$ in (2.1) with $\ell_{s}$, and re-arranging as in (2.2), I obtain the quadratic expression,

$$
\left(N^{-1 / 3} B o^{-1 / 2}\right)^{2}-\frac{1}{2} \frac{\sin \psi}{\sin \phi} H\left(N^{-1 / 3} B o^{-1 / 2}\right)+\frac{1}{\sin \psi \sin \phi}\left(\frac{1}{6} \cos ^{3} \psi-\frac{1}{2} \cos \psi-\frac{2}{3} \frac{D}{t}+\frac{1}{3}\right)=0 .
$$

The solution to (2.4) is in the form $N^{-1 / 3} B o^{-1 / 2}=\kappa^{-1 / 3}$, where the $O(1)$ pre-factor,

$$
\kappa^{-1 / 3}=\frac{1}{24} \frac{1}{\sin \psi \sin \phi}\left\{3 H-3 H \cos 2 \psi+2\left[-6(8-16 D / t-9 \cos \psi+\cos 3 \psi) \sin \psi \sin \phi+9 H^{2} \sin ^{4} \psi\right]^{1 / 2}\right\} .
$$

Thus, the expression for the stacking geometry's critical sphere number,

$$
N=\kappa B o^{-3 / 2}
$$

Notably, I find that in the stacking geometry, the critical sphere number, $N \propto B o^{-3 / 2}$, for all values of the Bond number $B o$, regardless of whether interfacial tension or buoyancy dominates the restorative force.

In Fig. 2.4 (b), I calculate $\kappa \approx 3.1$ by ensuring that the stacking geometry's model, $N=\kappa B o^{-3 / 2}$, converges to the raft geometry's expression, $N=\alpha B o^{-2}+\beta B o^{-1}$, when the critical sphere number $N=1$ (since the two modes of sphere deposition must be identical when there is just one sphere). The data from my experiments show a good agreement with the stack scaling $N=\kappa B o^{-3 / 2}$.

I note that the stack data is biased towards the raft model at lower values of sphere number $N$, and the deviation is more pronounced in experiments where the interfacial tension is higher; for example, when $\gamma=24.9$ and $18.5 \mathrm{mN} / \mathrm{m}$, as indicated by empty circles and triangles, respectively, in Fig. 2.4 (b). 
I attribute this deviation to the tendency of stacks, at low sphere number $(N<4)$, to reorient into a raft configuration (see for example the third frame of Fig. 2.3 (c)).

I interpret this observation by the following hypothesis. When a new sphere is stacked on top of a forming sphere cluster, it may cause the cluster to rotate to a new equilibrium position, which occurs over time $t_{e q}$. When the critical sphere is added, the cluster accelerates and passes through the oil-water interface over time $t_{\gamma}$. If the ratio of stabilization time $t_{e q}$ to interface passage time $t_{\gamma}$ is greater than unity, $t_{e q} / t_{\gamma}>1$ (i.e. passage occurs quickly) then the cluster will remain in the stack orientation as it passes through the interface. However if $t_{e q} / t_{\gamma}<1$ (i.e. reorientation happens more rapidly than passage), then the spheres will reorient into a raft, $\ell_{s} \rightarrow \ell_{r}$, where $\ell_{r}>\ell_{s}$, consequently increasing the interfacial tension contribution supporting the cluster, and preventing the cluster from sinking through the interface.

This effect diminishes at higher sphere numbers, because there is a greater difference in the critical sphere number between rafts and stacks. At higher sphere numbers, my experimental data clearly shows that there is a distinctively different scaling of the critical sphere number $N$, for particle stacks compared to rafts.

\subsection{Conclusions}

These experimental results and scaling analyses show that, at above the threshold density ratio $D_{\text {max }}$, floating self-assembled interfacial rafts and stacks will eventually collapse and sink when they reach a critical size. The scaling dependence of the critical sphere number $N$ on the Bond number $B o$ is distinct between rafts and stacks: particle rafts collapse when the sphere number $N=\alpha B o^{-2}+\beta B o^{-1}$; particle stacks follow the scaling for the sphere number, $N=\kappa B o^{-3 / 2}$.

This different scaling law for particle stacks may have important engineering implications in situations where the floating assemblies on liquid-liquid interfaces are not monolayers. For example, a recent publication shows the clustering and coating of self-assembled magnetic microparticles in microfluidics [9]. Flow-focused microparticles assemble into clusters on the co-flowing interface between water and oil in a multi-layered fashion. Thus, the size control of these microfluidic coated particle clusters may be 
determined by a power law similar to the one I show for particle stacks - albeit with a magnetic Bond number to account for magnetic forces in the microfluidic system. 



\section{Chapter 3}

\section{Microfluidic magnetic self-assembly of microparticle clusters at an aqueous two-phase liquid-liquid interface}

\subsection{Abstract}

I present a microfluidic system that self-assembles microparticles into clusters at an aqueous two-phase liquid-liquid interface. The liquid-liquid interface is formed between converging flows of aqueous dextran and polyethylene glycol in a microfluidic cross-slot device. I control the size of the self-assembled particle clusters as they pass through the liquid-liquid interface by systematically varying the applied magnetic field gradient and interfacial tension of the liquid-liquid interface. I observe rich assembly dynamics, including the formation of particle clusters and magnetic chains. Upon cluster penetration across the liquid-liquid interface, I find instances where the fluid coating drains away, and where the clusters are completely encapsulated inside droplets. I find that the number of particles within a cluster increases with increasing interfacial tension, and decreasing magnetic field gradient. Finally, I empirically observe an inverse scaling of the number of particles within a cluster to the dimensionless magnetic Bond number. 


\subsection{Introduction}

The interface formed between immiscible liquid phases has numerous applications in microfluidic devices [41, 42]. Liquid-liquid interfaces have been used for separation processes [43, 44], particle synthesis techniques [45, 46], and are well-known for their use in self-assembly processes [47, 48].

Recently, microfluidic technologies have facilitated the fabrication and self-assembly of a variety of particle clusters. For example, spherical particle clusters can be formed in evaporating drops [49], and Janus particles formed with droplet microfluidics can be designed to self-assemble into highly repeatable cluster geometries $[50,51]$.

In addition to self-assembly, forcing particles and particle clusters through a liquid-liquid interface can be exploited to conformally coat the assemblies in a thin film of one of the two immisible fluid phases $[9,10,52]$. For example, self-assembly and conformal coating of microparticle clusters has been demonstrated on a co-flowing oil-water interface by Tsai et al. [9].

Despite recent interest in microfluidic self-assembly, there has not been a detailed study on the selfassembly process and the parameters that govern the final cluster size. Here, I demonstrate controlled self-assembly of paramagnetic microparticles on a liquid-liquid interface. Specifically, I control the number of particles within a particle cluster by systematically varying the strength of the applied magnetic field gradient, and the interfacial tension of the liquid-liquid interface.

Passing microparticles through an interface via magnetic forcing is possible when the interface between the liquid phases has an ultra-low interfacial tension [53]. For oil-water systems, ultra-low interfacial tension is achieved with the use of surfactants $[9,53]$. However, surfactants have practical limitations. For example, above the critical micelle concentration (CMC), additional surfactants do not go to the interface to reduce the interfacial tension, but instead form micelles in the bulk fluid phase [53].

Aqueous two-phase systems (ATPS) are formed by dissolving two incompatible polymers in water, which phase separate above a critical dissolved polymer concentration. ATPS naturally have an ultralow interfacial tension, that can be tuned by adjusting the dissolved polymer concentration [13]. There are numerous polymer combinations that exhibit this behavior, but the combination of PEG and DEX 
is one of the most extensively studied.

Here, the ATPS liquid-liquid interface is formed in an extensional flow, between converging flows of aqueous PEG and DEX. Extensional flows in the microfluidic cross-slot geometry have been used primarily to study the dynamics of single polymer molecules [54], bulk polymer rheology [55, 56], and the deformation of microcapsules in a flow field [57]. The stagnation point which is produced in this type of flow can also be used as a hydrodynamic trap [58, 59]. Here, I use the extensional flow in the microfluidic device to control the position of the liquid-liquid interface within the microchannel.

The main benefit of this self-assembly system is that the stagnation point, which forms on the liquidliquid interface as a result of the extensional flow, significantly reduces the speed of the fluid at the interface, and thus reduces the drag force experienced by the microparticles during the self-assembly process. Additionally, the extensional flow brings particles directly to the liquid-liquid interface, in comparison to a co-flow self-assembly system where the particles must be magnetically forced through the bulk fluid phase to reach the interface. Finally, the device forms a curved liquid-liquid interface, which drives the self-assembly process toward the apex of the curved interface, and thus produces larger cluster geometries than seen in the co-flow self-assembly geometry [9].

This chapter is organized as follows: I first report my experimental observations of the cross-flow microchannel geometry, and the interaction of individual microparticles with the liquid-liquid interface. I then present my observations of the self-assembly of microparticles, and their subsequent passage through the liquid-liquid interface. In particular, I investigate the number of particles within the selfassemblies, and whether they entrain fluid while passing through the liquid-liquid interface. I find that the number of particles within a cluster increases with increasing interfacial tension, and also increases when the strength of the magnetic field is decreased.

\subsection{Experimental Methods}

\subsubsection{Chemical preparation}

I prepare the ATPS by combining aqueous solutions of polyethylene glycol (PEG, Mw 35k, SigmaAldrich, St. Louis, MO, USA) and dextran (DEX, Mw 500k, Pharmacosmos, Holbaek, Denmark). The 
Table 3.1: Composition of the eight dextran-polyethylene glycol ATPS ${ }^{b}$

\begin{tabular}{llllll}
\hline ATPS & $\begin{array}{l}\text { PEG } \% \\
(\mathrm{w} / \mathrm{v})\end{array}$ & $\begin{array}{l}\text { DEX } \% \\
(\mathrm{w} / \mathrm{v})\end{array}$ & $\begin{array}{l}\eta_{p} \\
(\mathrm{mPa} \cdot \mathrm{s})\end{array}$ & $\begin{array}{l}\eta_{d} \\
(\mathrm{mPa} \cdot \mathrm{s})\end{array}$ & $\begin{array}{l}\gamma_{p d} \\
(\mathrm{mN} / \mathrm{m})\end{array}$ \\
\hline 1 & 5.0 & 6.4 & 5.1 & 14.7 & 0.012 \\
2 & 5.0 & 16.0 & 9.8 & 32.3 & 0.037 \\
3 & 5.0 & 20.0 & 12.6 & 50.2 & 0.042 \\
4 & 10.0 & 12.8 & 15.0 & 65.1 & 0.082 \\
5 & 10.0 & 16.0 & 16.4 & 67.5 & 0.103 \\
6 & 10.0 & 20.0 & 28.0 & 153.3 & 0.150 \\
7 & 15.0 & 19.2 & 39.1 & 248.7 & 0.209 \\
8 & 20.0 & 25.6 & 89.3 & 713.9 & 0.381 \\
\hline
\end{tabular}

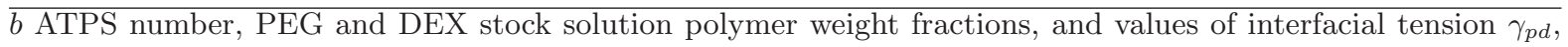
are from Atefi et al. [13].

polymer concentration and fluid properties of the eight ATPS used in my experiments is summarized in Table 3.1. The preparation procedure is based of the methodology of Atefi et al. [13].

For each ATPS, a pair of $100 \mathrm{~mL}$ stock solutions of PEG and DEX is prepared, where the stock solutions consist of $5.0-20.0 \%$ (w/v) PEG and $6.4-25.6 \%$ (w/v) DEX, dissolved in deionized (DI) water. Once completely mixed, each pair of PEG and DEX stock solutions are combined, vigorously mixed, and left for $>24$ hours to completely separate. The high-density DEX-rich phase is then separated from the low-density PEG-rich phase with syringes (BD Medical, Franklin Lakes, NJ, USA). I use a glass viscometer to measure the DEX and PEG phase viscosities, $\eta_{d}$ and $\eta_{p}$, respectively, and I use the interfacial tensions, $\gamma_{p d}$, measured by Atefi et al. [13].

I use polystyrene paramagnetic microparticles that have radius $a=5 \mu \mathrm{m}$ (Sigma-Aldrich, St. Louis, MO, USA) in my experiments. $100 \mu \mathrm{L}$ of the stock microparticle solution (5\% solid concentration) is added to $1 \mathrm{~mL}$ of the DEX phase, and thoroughly mixed with a vortex mixer. The particle suspension is then flash centrifuged in a conical vial, and the carrier liquid is removed with a pipette. The washed microparticle pellet is then resuspended in $1 \mathrm{~mL}$ of DEX, and loaded into a $1 \mathrm{~mL}$ syringe for the experiments.

\subsubsection{Device fabrication}

The microfluidic chip is fabricated with a layer of polydimethylsiloxane (PDMS, Sylgard 184, Dow Corning, Midland, MI, USA), patterned with the standard soft lithography technique [60,61]. The 
microchannel geometry is drawn with computer-aided design (CAD) software and printed onto a transparency sheet (25,400 dpi, CAD/ART Services Inc., Bandon, OR, USA) to form a photomask. I spin-coat SU-8 2025 negative photoresist (Microchem., Newton, MA) onto a 4-inch silicon wafer, and expose the substrate to UV light through the photomask. After the silicon wafer is developed, the PDMS channels are formed by pouring PDMS (10:1 prepolymer to curing agent) over the silicon wafer, which is then cured in an oven, which produces a microchannel with a height $h=50 \mu \mathrm{m}$. The edge of the patterned

PDMS layer is trimmed with a straight razor (Personna, Verona, VA, USA), to allow the placement of a permanent magnet in close proximity $(<1 \mathrm{~mm})$ to the cross-slot region of the microchannel. Inlet and outlet holes are punched into the patterned PDMS layer with a 1 diameter mm biopsy punch (Integra Miltex, Inc., Rietheim-Weilheim, Germany). The patterned PDMS layer is then permanently bonded via oxygen plasma treatment (Harrick Plasma, Ithaca, NY, USA) to a glass cover slip $(50 \times 22 \times 0.2$ mm, Thermo Fisher Scientific Inc., MA, USA) with the cross-flow region of the PDMS layer placed at the outside edge of the glass slide (see Figs. 3.1 (a) and (b)).

\subsubsection{Experimental setup}

A neodymium boron magnet (NdFeB, B22X0, K. J. Magnetics, Jamison, PA, USA) with magnetizaton $M \approx 1.05 \mathrm{MA} / \mathrm{m}$ is used to provide the magnetic field gradient, and is placed in close proximity to the cross-junction of the microchannel (Fig. 3.1 (b)). A 3D printed fixture is used to control the alignment of the microfluidic chip and the permanent magnet. As seen in Figure 3.1 (b), the magnet is secured to a glass slide to allow repeatable alignment of the magnet. The magnet is positioned such that the center of the magnet face is aligned with the cross-flow region of the microchannel, to reduce the vertical and lateral components of the magnetic field gradient.

The microparticle suspension and the two aqueous phases are pumped with syringe pumps (Harvard Apparatus, Holliston, MA, USA), through polyethylene tubing (Instech Laboratories, Inc., PA, USA), to the corresponding inlets of the microfluidic device. Syringes are interfaced with the tubing via blunt needle syringe tips (Fishman Corporation, MA, USA). The syringe pump that controls the particle suspension flow rate is positioned vertically above the microfluidic chip. This setup reduces variations in the particle flux into the microfluidic device from particles sedimenting in the tubing. Additionally, 


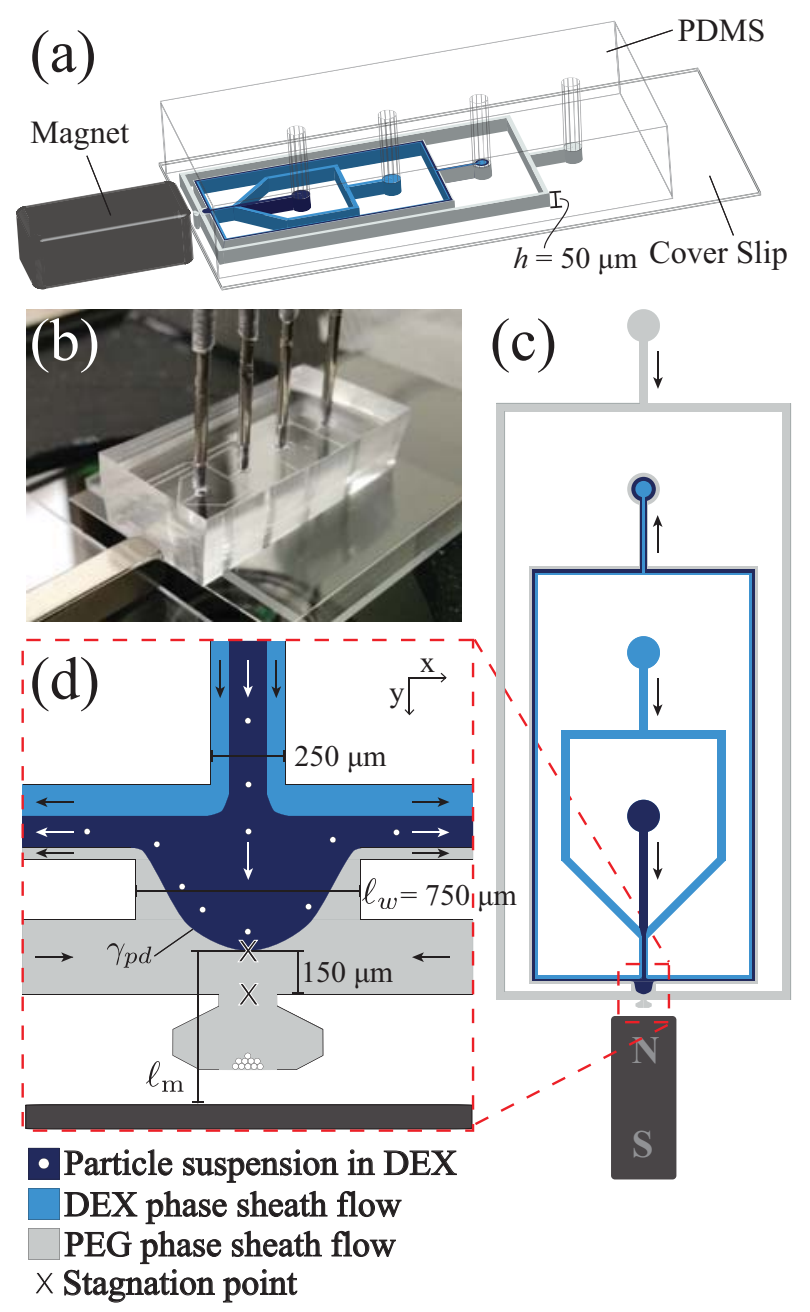

Figure 3.1: (a) Schematic diagram of the microfluidic chip. A patterned layer of PDMS is plasma-bonded to a glass cover slip. A permanent magnet is placed at the edge of the microfluidic chip to provide the magnetic field. (b) Experimental set-up of the of microfluidic system with attached inlet and outlet ports. The microfluidic chip and permanent magnet are aligned on a 3D printed fixture, where the face of the magnet is centered on the cross-slot region of the microchannel. (c) Top view of the microchannel system. Paramagnetic microparticles suspended in the DEX solution are injected into the channel, and flow-focused by a DEX phase sheath flow. The total DEX phase flow converges with the PEG phase to form a stable curved liquid-liquid interface in the cross-slot chamber. Arrows indicate the direction of flow. (d) An expanded view of the cross-slot chamber shows the curved liquid-liquid interface formed from converging PEG and DEX flows. Stagnation points, shown by the symbol $\times$ are formed at the apex of the liquid-liquid interface, and at the throat of the cluster collection chamber. Particles that enter the cross-slot chamber at the center of the DEX-rich flow reach the apex of the liquid-liquid interface, and may pass through the interface into the PEG phase collection chamber. 
the microparticle suspension is remixed just prior to each experiment to ensure a homogeneous mixture.

Figure 3.1 (c) shows a top view of the mirochannels in the PDMS device. Paramagnetic microparticles suspended in the DEX phase are injected into the microfluidic device, where they are flow-focused by a DEX phase sheath flow. The flow focused DEX phase converges with the PEG phase in the cross-slot chamber to form a stable liquid-liquid interface. The combined PEG and DEX phases then flow into symmetrical outlets in an extensional flow, which stabilizes the liquid-liquid interface. Figure 3.1 (d) shows a magnified schematic diagram of the microfluidic cross-slot, where particles suspended in the DEX phase flow to the liquid-liquid interface. Stagnation points are formed at the apex of the liquidliquid interface, and at the throat of the collection chamber in the PEG T-junction (marked with $\times$ in Figure $3.1(\mathrm{~d}))$.

The flow rate of the microparticle suspension and the DEX phase sheath flow are each set to $2 \mu \mathrm{L} / \mathrm{min}$, for a total DEX phase flow rate $Q_{d}=4 \mu \mathrm{L} / \mathrm{min}$. The PEG phase flow rate is set to $Q_{p}=2 \mu \mathrm{L} / \mathrm{min}$, and adjusted to maintain the distance between the apex of the liquid-liquid interface and the inner chamber wall of $150 \mu \mathrm{m}$ (see Fig 3.1 (d))). Due to the varied viscosity ratio of the PEG and DEX phases for different ATPS compositions, small adjustments in the PEG flow rate $\left(Q_{p}=1.8-4.4 \mu \mathrm{L} / \mathrm{min}\right)$ are required to achieve the same interface location, $\ell_{m}$, in the microfluidic cross-junction for each ATPS composition.

Experimental images are captured by an inverted microscope (IX71, Olympus Corp., Tokyo, Japan) with a 20x objective, and a high speed camera (Miro M110, Vision Research, Wayne, NJ, USA) at a frame rate of $100 \mathrm{fps}$, and an exposure time of 1000 ps. High resolution images are taken with a 50x objective, at a frame rate of $200 \mathrm{fps}$, and an exposure time of $500 \mu \mathrm{s}$. Image $\mathrm{J}$ is used for image processing [62], where particle trajectory images were compiled with the minimum intensity Z-projection function which saves the lowest pixel value (black $=0$, white $=255$ ), where the opaque particles appear black, and the translucent PDMS and glass background appears white. 


\subsection{Results and Discussion}

\subsubsection{Flow in the microfluidic cross-slot}

Under flow conditions, a cross-slot microchannel geometry produces an extensional flow field, with a stagnation point at the center of the converging flows $[55,56]$. I utilize this type of extensional flow to control the position of the liquid-liquid interface in my experiments. The two aqueous phases converge in the cross-slot chamber to form the liquid-liquid interface, and results in a stagnation point at the center of the liquid-liquid interface (see Fig. $3.1(\mathrm{~d})$ ). The combined PEG and DEX phases then flow in a co-flow configuration into the symmetrical side channels.

To facilitate the placement of a permanent magnet in close proximity $(<1 \mathrm{~mm})$ to the liquid-liquid interface, I modify the geometry of the inlets to the cross-slot chamber. Typical cross-slot channel geometries are both horizontally and vertically symmetric $[54,55,59,63]$. In my design, the PEG phase inlet splits into two channels, and merges back into a single channel in a T-junction, prior to arriving at the cross-slot junction (see Fig. 3.1 (c)). As a result of this T-junction, a second stagnation point is formed at the throat of the PEG collection chamber [58], in addition to the stagnation point at the center of the converging flows of PEG and DEX (see Fig. $3.1(\mathrm{~d})$ ). I also expand the width of the microchannel at the cross-slot junction, from $250 \mu \mathrm{m}$ to $\ell_{w}=750 \mu \mathrm{m}$ at the junction. As a result of the modified channel geometry, the liquid-liquid interface has a curved shape, with the stagnation point located at the apex of the curved interface.

\subsubsection{Single particles at the liquid-liquid interface}

Microparticles suspended in the DEX phase flow with the bulk fluid into the cross-flow chamber. In the absence of a magnetic field gradient, particles follow the fluid streamlines of the bulk fluid phase. As shown in the schematic diagram of Fig. $3.1(\mathrm{~d})$, particles in the center of the DEX phase flow to the liquid-liquid interface and reach a stagnation point, whereas particles which are not flow-focused to the center will flow through the periphery of the cross-flow chamber to the side channels without reaching the liquid-liquid interface. As a result of the stagnation point at the apex of the liquid-liquid interface, and the increased cross-sectional area of the cross-slot chamber, I observe that microparticles that enter 
the cross-slot chamber slow down significantly as they approach the liquid-liquid interface. Still, without the presence of a magnetic field gradient, the particle will be washed to one of the symmetric fluid outlets due to hydrodynamic shear stresses from the moving fluid.

When I apply the magnetic field gradient, the paramagnetic particles flowing into the cross-flow chamber will deflect from the bulk fluid streamlines towards the liquid-liquid interface. Microparticles that reach the interface often become adsorbed into the interface, forming a three-phase contact line between the two ATPS phases and the particle surface. This particle adsorption is characterized by the "snap-in" effect, where the particle rapidly accelerates into the interface at the instant that the drainage film ruptures and the three-phase contact line is formed [44]. Subsequently, these particles detach from the interface, and pass into the PEG phase. This type of particle passage is analogous to the drainage regime in the fluid mechanics literature $[64,65]$. Figure 3.2 (a) shows an experimental image of a particle passing through the interface in the drainage regime. The DEX fluid film between the particle and the liquid-liquid interface completely drains away at $t \approx 0.2 \mathrm{~s}$, where the 3 -phase contact line is formed. The particle subsequently detaches from the interface at $t \approx 1.15 \mathrm{~s}$, and passes into the PEG phase. The film drainage process is gradual, so I also observe instances where particles become adsorbed into the liquid-liquid interface, and do not detach from the interface before being washed into one of the side channels.

When the magnetic body force acting on the particle is sufficiently large that it overcomes the restoring force from the liquid-liquid interface, the particle passes from the DEX phase, through the liquid-liquid interface into the PEG phase, without becoming adsorbed into the interface. Particles that pass through the liquid-liquid interface in this manner entrain a volume of the DEX phase as they pass through the interface, which forms an interfacial tail behind the particle as it moves through the PEG phase. This type of particle passage is analogous to the tailing regime in the fluid mechanics literature $[64,65]$. Figure 3.2 (b) shows an example of microparticle passing through the interface in the tailing regime, where the particle entrains some of the DEX phase and forms the interfacial tail upon passing through the liquid-liquid interface. 

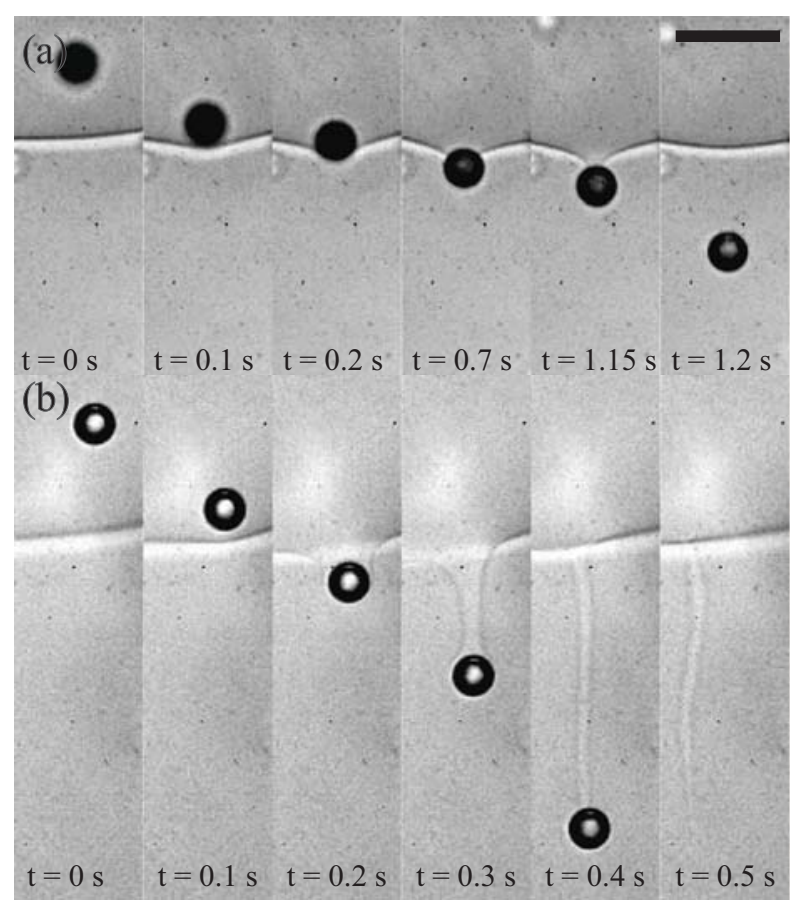

Figure 3.2: Experimental images of a single microparticle passing through the liquid-liquid interface in the (a) drainage regime, where the DEX fluid film must completely drain away, before the particle is able to pass through the interface. Here the 3 -phase contact line forms at $t=0.2 \mathrm{~s}$, where the particle adsorbs into the interface. The particle detaches from the interface at $t=1.15 \mathrm{~s}$, after which it passes into the PEG phase. Here, interfacial tension $\gamma_{p d}=0.150 \mathrm{mN} / \mathrm{m}$. In contrast, a single particle passing through in the interface in the (b) tailing regime. As the particle approaches the liquid-liquid interface it causes the interface to deform, but the particle is able to pass through the interface without forming a 3-phase contact line. As the particle continues into the PEG phase, it entrains some of the DEX phase in an interfacial tail. Here, the interfacial tension $\gamma_{p d}=0.012 \mathrm{mN} / \mathrm{m}$. Scale bar indicates $25 \mu \mathrm{m}$. 


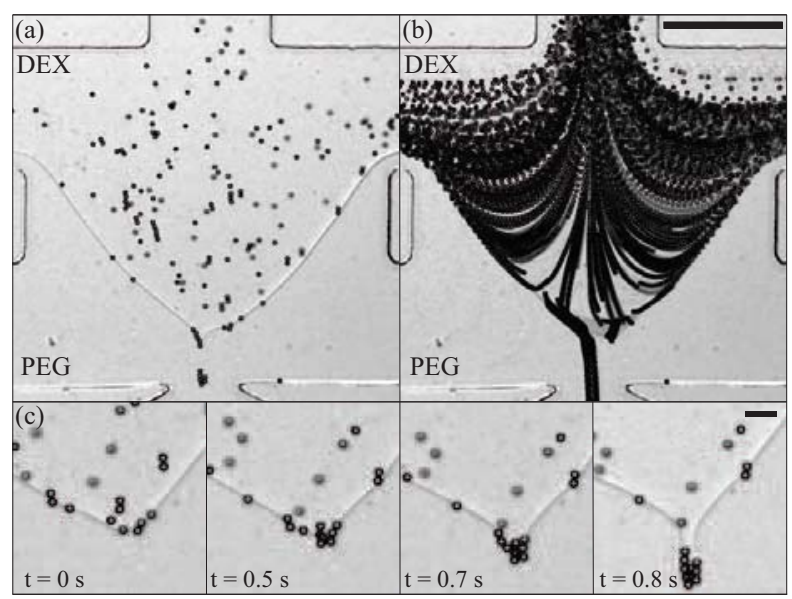

Figure 3.3: Experimental images of (a) the cross-flow chamber during the magnetic cluster self-assembly process, (b) the particle trajectories during self-assembly. Scale bar indicates $250 \mu \mathrm{m}$. (c) A close-up view of a microparticle cluster forming on the liquid-liquid interface, where the cluster with particle number $N=9$ is formed on an interface with interfacial tension $\gamma_{p d}=0.103 \mathrm{mN} / \mathrm{m}$. Scale bar indicates $50 \mu \mathrm{m}$.

\subsubsection{Self-assembly at the liquid-liquid interface}

I observe that microparticles that reach the apex of the liquid-liquid interface self-assemble into clusters due to the local magnetic field gradient provided by the permanent magnet. If the self-assembled particle cluster is sufficiently large, it will pass through the liquid-liquid interface into the PEG phase. Figure 3.3 (a) shows an experimental image the cross-slot chamber during the extensional flow and self-assembly process. Here, particles enter the chamber from the upper DEX inlet, and flow towards the liquid-liquid interface. In this case the microparticles assemble into a cluster with particle number $N=8$, which then passes through the liquid-liquid interface into the PEG phase, and collects in the PEG phase collection chamber.

Figure 3.3 (b) shows the trajectories of microparticles as they flow through the cross-slot chamber in the presence of a magnetic field. Microparticles in the center of the DEX phase flow directly to the apex of the liquid-liquid interface, where the particles are able to self-assemble into a cluster. Particles that are further from the center of the channel when they enter the cross-slot chamber are deflected away from the centerline by the bulk fluid flow, but are often still able to reach the liquid-liquid interface. 
Some of the particles that reach the interface will move towards the apex of the liquid-liquid interface and will self-assemble, whereas the particles that reach the interface further from the stagnation point will eventually flow with the bulk fluid to one of the symmetrical outlets. Figure 3.3 (c) shows time series images of the particle cluster as it self-assembles on the liquid-liquid interface, and passes through to the PEG phase.

\subsubsection{Different types of self-assembled clusters}

I observe rich self-assembly dynamics, due to the combination of the interfacial effects from the presence of the liquid-liquid interface, magnetic effects from the magnetic field, and hydrodynamic forces from the extensional flow field. I classify the types of self-assembly into the following three categories: individual interfacial self-assembly, magnetic self-assembly, and combined interfacial self-assembly (see Fig. 3.4 (a), (b), and (c), respectively). Individual interfacial self-assembly refers to clusters formed from individual particles, which assemble exclusively on the liquid-liquid interface, prior to passing through the liquidliquid interface to the PEG phase (see Fig. 3.4 (a)). Magnetic assembly refers to particles that assemble into a chain via dipole-dipole interactions prior to interaction with the interface, and pass through the interface without any interaction with additional particles at the liquid-liquid interface (for example Fig. 3.4 (b)). Combined interfacial self-assembly also refers to the self-assembly of particles on the interface, but it includes instances where particle chains are formed prior to the interface, and are assembled into larger clusters at the liquid-liquid interface (see Fig. 3.4 (c)).

In all of my experiments, I observe that in a magnetic field, paramagnetic particles in close proximity to each other (approximately one particle radius) are able to spontaneously align into chains, due to dipole-dipole interactions. I observe this behavior most frequently with two individual particles aligning into a doublet, but particle chains with a larger number of particles are also possible. I observe that the formation of particle chains frequently occurs as particles enter the cross-slot chamber, due to the decreased inter-particle distances as a result of the reduced particle speed, and the increased strength of the magnetic field as the particles flow closer to the magnet.

If the liquid-liquid interfacial tension $\gamma_{p d}$ is sufficiently low, microparticle chains formed prior to the interface are able to pass directly though the interface in the tailing regime. As I gradually raise 


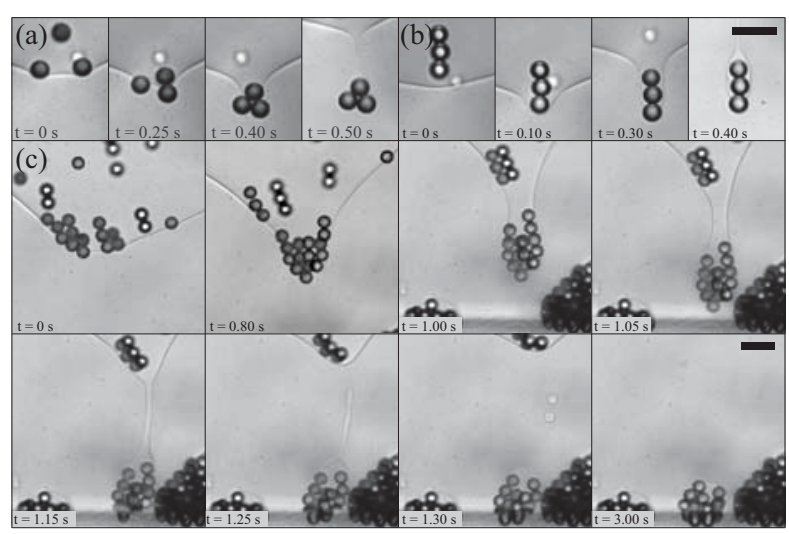

Figure 3.4: Experimental images of microparticle clusters passing through the liquid-liquid interface. I differentiate between (a) individual interfacial self-assembly, where individual microparticles assemble at the liquid-liquid interface, and (b) magnetic self-assembly, where individual microparticles assemble into chains, via magnetic dipole-dipole interactions, prior to reaching the interface. Here, the liquid-liquid interfacial tension $\gamma_{p d}=0.103 \mathrm{mN} / \mathrm{m}$. (c) If the interfacial tension $\gamma_{p d}$ is sufficiently large, I observe a combination of interfacial and magnetic self-assembly, where small particle chains assemble into a larger particle clusters at the liquid-liquid interface. I call this combined interfacial self-assembly. Here the interfacial tension $\gamma_{p d}=0.209 \mathrm{mN} / \mathrm{m}$. Scale bars indicate $25 \mu \mathrm{m}$.

the interfacial tension $\gamma_{p d}$, smaller particle chains, such as doublets, are not able to pass through the interface. Instead, these chains self-assemble with other particles into larger clusters.

Figure 3.5 shows the frequency of magnetic self-assembly in comparison to interfacial self-assembly (both individual and combined) for each combination of ATPS composition and magnet distance. Despite the prevalence of dipole-dipole chain formation in my experiments, I observe that the liquid-liquid interface plays a crucial role in the self-assembly of microparticle clusters. I observe the formation of magnetic chains due to dipole-dipole interactions, prior to the liquid-liquid interface, in all of my experiments. However, in most cases these chains are assembled via combined self-assembly into larger particle clusters.

\subsubsection{Controlling the size of self-assembled clusters}

I form particle clusters on a liquid-liquid interface, and control the number of particles $N$ within individual clusters by systematically varying the location of the magnet $\ell_{m}$, and by adjusting liquid-liquid 

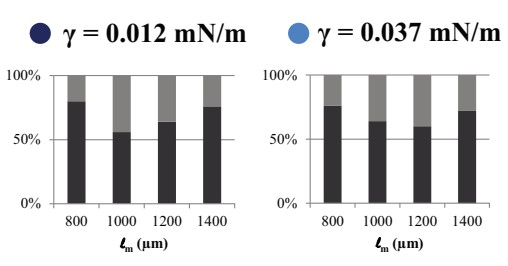

$\gamma=0.042 \mathrm{mN} / \mathrm{m}$

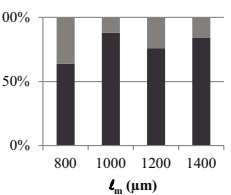

$\gamma=0.082 \mathrm{mN} / \mathrm{m}$

$\gamma=0.103 \mathrm{mN} / \mathrm{m}$

$\gamma=0.150 \mathrm{mN} / \mathrm{m}$
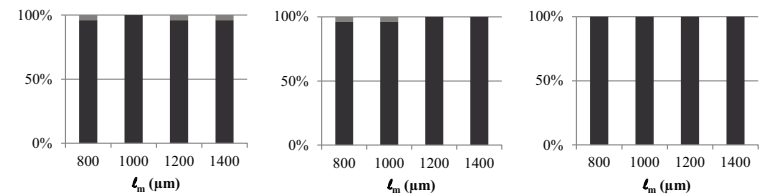

$\gamma=0.209 \mathrm{mN} / \mathrm{m}$

$\gamma=0.381 \mathrm{mN} / \mathrm{m}$
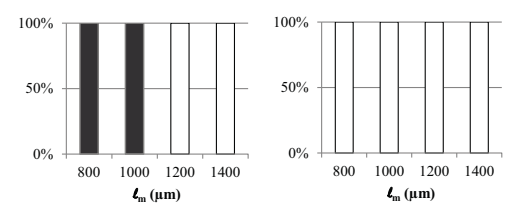

Interfacial self-assembly

$\square$ Magnetic self-assembly

$\square$ No cluster passage

Figure 3.5: Stacked bar graphs showing the percentage of each self-assembly type, versus magnet distance $\ell_{m}$. Interfacial self-assembly and magnetic self-assembly are indicated by black and grey bars, respectively, and white bars show instances where none of the self-assembled clusters were able to pass through the interface. The results from each set of magnet distance $\ell_{m}$ and interfacial tension $\gamma_{p d}$ are from the 25 clusters which pass through the liquid-liquid interface. 


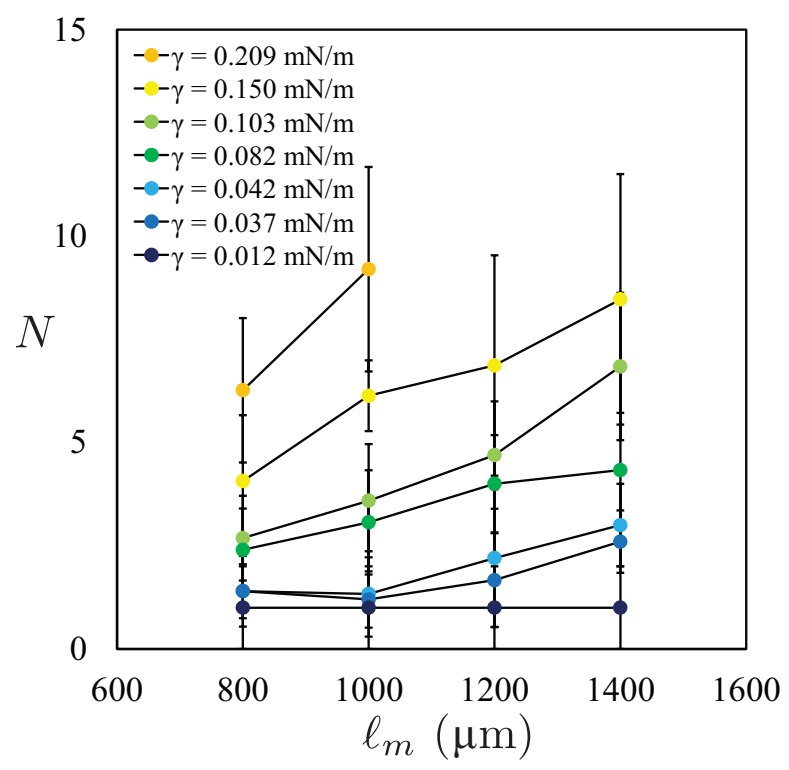

Figure 3.6: A plot of the cluster particle number $N$ versus magnet position $\ell_{m}$, where $\ell_{m}$ is the distance between the magnet face and the apex of the liquid-liquid interface. The interfacial tension $\gamma_{p d}$ of the liquid-liquid interface is varied by adjusting the dissolved polymer concentration of the ATPS (summarized in Table 1). I observe that particle number $N$ increases with magnet distance $\ell_{m}$, and also grows with interfacial tension $\gamma_{p d}$. Error bars represent one standard deviation.

interfacial tension $\gamma_{p d}$. Figure 3.6 shows a plot of the cluster particle number $N$ versus the magnet distance $\ell_{m}$ for cases of interfacial self-assembly, produced from the same dataset as Figure 3.5, where each point represents the average of the first 15 samples. Here I have included only clusters which were formed with the influence of the liquid-liquid interface, that is both individual and combined interfacial self-assembly, but not magnetic self-assembly. I observe that as I increase the magnet distance $\ell_{m}$, which reduces the strength of the magnetic field, a greater number of particles $N$ is required to overcome the interfacial tension energy barrier and pass through the interface. I also modify the interfacial tension $\gamma_{p d}$ of the liquid-liquid interface by adjusting the ATPS polymer concentration (see Table 3.1), to see how this affects the self-assembly process. I observe an approximately monotonic trend of the cluster particle number $N$ with increasing magnet distance, and with increasing interfacial tension $\gamma_{p d}$.

I examine the competition of the magnetic body forces driving the self-assembly process, to the viscous drag, and to the interfacial tension restoring forces. In my experiments I measure a maximum 
Reynolds number $R e \approx O\left(10^{-4}\right)$, and thus I neglect the effects of inertia in my analysis. I first consider the competition of the magnetic force $\boldsymbol{F}_{m}$ and the viscous drag $\boldsymbol{F}_{d}$ acting on a particle as it approaches the liquid-liquid interface. Here the magnetic force is [53],

$$
\boldsymbol{F}_{m}=4 \pi a^{3} \mu_{o} \frac{\chi}{\chi+3} \nabla \boldsymbol{H}^{2}
$$

where the magnetic field is $\boldsymbol{H}$, the particle magnetic susceptibility $\chi$ is the dimensionless constant that indicates the degree of the particle magnetization in response to an applied magnetic field, where $\chi \approx$ $10^{-3}$, and the permeability of free space $\mu_{o}=1.257 \times 10^{-6} \mathrm{~m} \mathrm{~kg} \mathrm{~s}^{-2} \mathrm{~A}^{-2}$. In addition I note that $(\chi / \chi+3) \rightarrow \chi$ for $\chi<<1$. The viscous drag is,

$$
\boldsymbol{F}_{d}=-6 \pi \eta_{d} a \boldsymbol{v}
$$

where $\boldsymbol{v}$ is the characteristic velocity of the microparticle. Here I consider the force balance on the microparticle in the y-direction (see Fig. 3.1), prior to the interaction with the liquid-liquid interface, where there is a balance between the magnetic force acting on the particle and the viscous drag, $\boldsymbol{F}_{m}+$ $\boldsymbol{F}_{d}=0$. I non-dimensionalize the magnetic field $\hat{H}=\boldsymbol{H} / M$, the length scale $\hat{y}=y / \ell_{m}$, and the characteristic velocity $\hat{v}=\boldsymbol{v}_{y}\left(\ell_{w} h / Q_{d}\right)$, and the force balance becomes,

$$
0=4 \pi a^{3} \mu_{o} \chi \frac{M^{2}}{\ell_{m}}\left(\frac{\partial \hat{H}^{2}}{\partial \hat{y}}\right)-6 \pi \eta_{d} a \frac{Q_{d}}{\ell_{w} h} \hat{v}_{y}
$$

where $\partial \hat{H}^{2} / \partial \hat{y}$ and $\hat{v}_{y}$ are $\mathrm{O}(1)$ terms. The competition of magnetism and viscosity acting on the magnetic particle is thus a function of the Mason number $M n$, the dimensionless ratio of viscous drag to magnetic effects,

$$
M n=\frac{\eta_{d} Q_{d} \ell_{m}}{a^{2} M^{2} \chi \mu_{o} h \ell_{w}}
$$

Furthermore, I examine the Capillary number $C a$, the dimensionless ratio of viscous effects to surface tension, where, 


$$
C a=\frac{\eta_{p} Q_{p}}{\gamma_{p d} h \ell_{w}}
$$

In my experiments I observe that when the magnet distance $\ell_{m}$ is increased, a greater number of particles $N$ is required for a self-assembled cluster to pass through the liquid-liquid interface. Similarly, when I increase the liquid-liquid interfacial tension $\gamma_{p d}$, a greater number of particles is required for the cluster to pass through the interface. Thus I group these terms together to form a single dimensionless parameter,

$$
C a \cdot M n^{-1}=\frac{Q_{p} \eta_{p}}{Q_{d} \eta_{d}} \frac{a}{\ell_{m}} \frac{a M^{2} \chi \mu_{o}}{\gamma_{p d}},
$$

Figure 3.7 shows a log-log plot of the cluster particle number $N$ versus the dimensionless term $\left(Q_{p} \eta_{p} / Q_{d} \eta_{d}\right)\left(a / \ell_{m}\right)\left(a M^{2} \chi \mu_{o} / \gamma_{p d}\right)$, for cases of interfacial self-assembly. Here, I have included points where the average cluster size $N>1$, that is where the presence of the interface causes particles to selfassemble into clusters. I observe empirically that the number of particles $N$ within a cluster is inversely proportional to the dimensionless term $\left(Q_{p} \eta_{p} / Q_{d} \eta_{d}\right)\left(a / \ell_{m}\right)\left(a M^{2} \chi \mu_{o} / \gamma_{p d}\right)$.

In addition, in my experiments as I increase the total polymer concentration of the ATPS solution, I observe a decreased PEG:DEX viscosity ratio, $\eta_{p} / \eta_{d}$ (See Table 3.1). In order to achieve the same interface location in the cross-flow chamber, I correspondingly increase the flow rate ratio $Q_{p} / Q_{d}$. I observe that the dimensionless ratio $Q_{p} \eta_{p} / Q_{d} \eta_{d} \approx 0.14$ for all of my experiments. I thus treat the dimensionless ratio $Q_{p} \eta_{p} / Q_{d} \eta_{d}$ as a constant, and incorporate it into the prefactor $\kappa^{\prime}$, where $\kappa^{\prime}=$ $\kappa Q_{p} \eta_{p} / Q_{d} \eta_{d}$. This reduces the dimensionless term to,

$$
N=\kappa^{\prime}\left(\frac{a}{\ell_{m}} \frac{a M^{2} \chi \mu_{o}}{\gamma_{p d}}\right)^{-1}=\kappa^{\prime}\left(\varepsilon B o_{m}\right)^{-1},
$$

where the prefactor $\kappa^{\prime} \approx 1.4$. The solid line in Figure 3.7 indicates the model $N=\kappa^{\prime}\left(\varepsilon B o_{m}\right)^{-1}$, where I see a good agreement between the empirical model and my experimental results. 


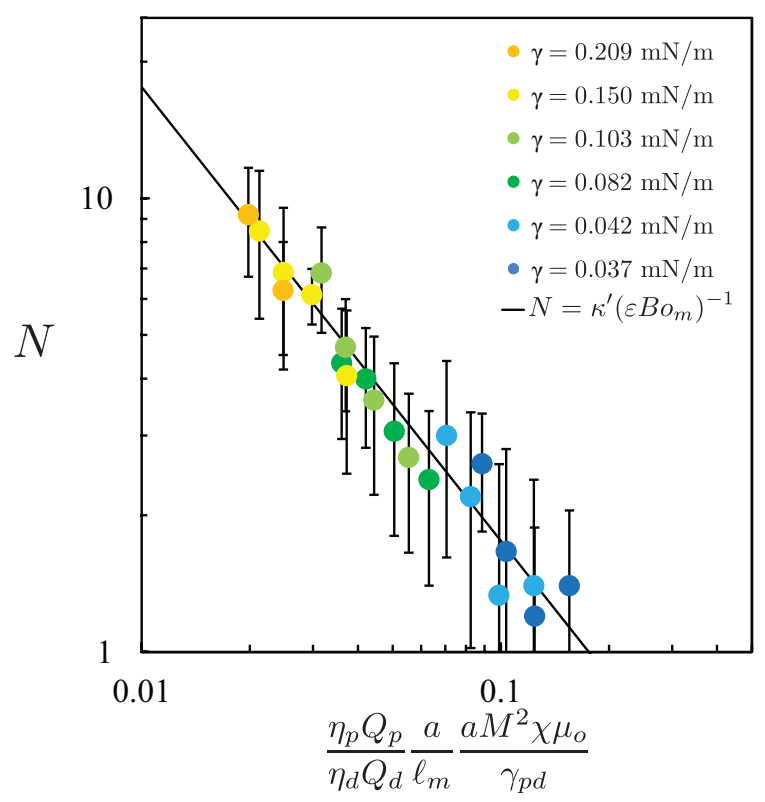

Figure 3.7: Log-log plot of the cluster particle number $N$, against the ratio of the Capillary and Mason numbers, $\mathrm{Ca} \cdot M n^{-1}$. I observe empirically that the particle number scales as $N=\kappa^{\prime}\left(\varepsilon B o_{m}\right)^{-1}$. Error bars represent one standard deviation.

\subsubsection{Coating microparticle clusters}

I observe that in some situations, particle clusters which pass through the liquid-liquid interface entrain a volume of the DEX phase as they pass into the PEG phase. Figure 3.8 shows a regime map of the fluid entrainment for single particles, double particle chains, and triple particle chains. I plot each selfassembly experiment on a log-log plot of Mason number $M n$, versus the Capillary number $C a$. Solid circles represent the tailing regime, where particles pass through the interface and entrain some of the DEX phase. Open circles represent the drainage regime, where particles are able to pass through the interface, but all of the DEX phase drains away prior to the particles passing through the interface. Crosses represent instances where particles are not able to pass through the interface.

Fig. 3.8 (a) shows the regime map for single particles which are forced through a liquid-liquid interface. Here dashed lines indicate the approximate boundaries between the tailing and drainage regime, and the drainage and no passage regime. If Mason number is large (i.e. there is a large magnetic component), microparticles pass through the liquid-liquid interface without forming a 3-phase contact 


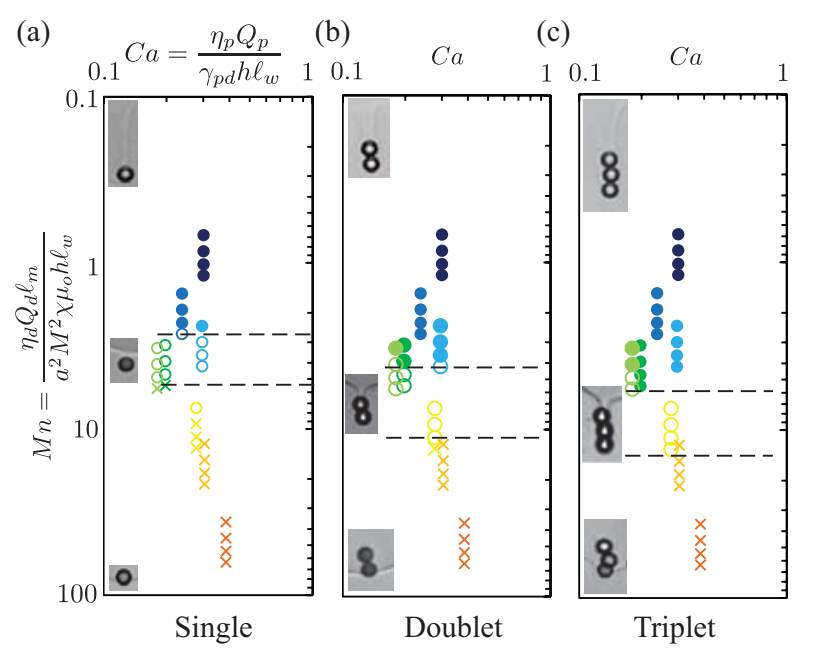

Figure 3.8: Regime map for (a) single particles, (b) doublets, and (c) triplets. Here solid circles represent the tailing regime, open circles indicate the drainage regime, and crosses indicate no passage. Marker color corresponds to the value of interfacial tension. I observe that when the Mason number is small, particles pass through the interface in the tailing regime, which is characterized entrainment of the DEX phase with the particle cluster. When Mason number is large, particles pass though the interface in the drainage regime, which is characterized by the complete drainage of the DEX phase liquid film from the particle surface. Here, the particles are able to pass through the interface, but are not encapsulated in a coating of the upper DEX phase. When the Mason number is very large, the particles are not able to pass through the liquid-liquid interface. Dash lines indicate the approximate boundaries between the tailing and drainage regimes, and the drainage and no passage regimes. 
line, and passes into the PEG phase while retaining a coating layer of DEX and forming an interfacial tail. As the particle continues into the PEG phase, the interfacial tail thins and eventually ruptures. As I reduce the Mason number, particles which reach the interface are adsorbed into the interface, and form the 3-phase contact line. These particles are able to detach from the interface to pass into the PEG phase, but do not become coated in the DEX phase.

Figure 3.8 (b) and (c) show the regime map for double particle chains and triple particle chains, respectively. Similar to single particles, I observe that when Mason number is large, particle chains pass through the liquid-liquid interface in the tailing regime, and entrain a volume of the DEX phase. In the drainage regime, the DEX fluid layer completely drains away before the particles pass through the interface. I note that near the boundary between the drainage regime and the tailing regime, particles at the front chain will form a 3-phase contact line with the liquid-liquid interface, but the chain is able to pass through the interface before the fluid around the chain completely drains away. As a result, the chain does entrain a volume of the DEX phase, however is not completely encapsulated in the DEX phase. Instead the particles are partially exposed to the PEG phase at the front of the chain.

For particle clusters with more than three particles, I observe that a volume of the DEX phase becomes trapped in between the particles within the cluster, and is not able to completely drain away. In some cases, due to the tendency of microparticles to become adsorbed into the liquid-liquid interface, particle clusters are not perfectly conformally coated in the DEX fluid phase (see for example Fig 3.4 (c) at $t=3.0$ seconds). In these cases, the particles in the center of the cluster are encapsulated within the DEX phase droplet, but the particles at the edge of the cluster may be partially exposed to the PEG phase.

\subsection{Conclusions}

In this chapter, I have demonstrated the self-assembly of paramagnetic microparticles into clusters on a liquid-liquid interface. I show that the number of particles within a cluster can be tuned by adjusting the interfacial tension $\gamma_{p d}$ of the liquid-liquid interface, or by adjusting the strength of the magnetic field. I

find that the number of particles $N$ within a cluster scales empirically with $\kappa^{\prime}\left(\varepsilon B o_{m}\right)^{-1}$. I also observe 
that in the small Mason number regime, particle clusters that pass through the liquid-liquid interface entrain a volume of the DEX fluid phase. This system could be applied to form coated clusters of other paramagnetic materials, but could be particularly well suited for forming clusters of magnetized cells. This is due primarily to the natural biocompatability of the two aqueous phases, and the wide variety of functionalized magnetic particles available. 



\section{Chapter 4}

\section{Concluding Remarks}

In this thesis I have studied the self-assembly of spherical particles on liquid-liquid interfaces. I have found that the size of the self-assemblies when they pass through the interface can be controlled by adjusting the liquid-liquid interfacial tension, or the magnitude of the body force acting on the selfassembly.

In the millimeter length scale, I found that the number of particles within a cluster is proportional to the dimensionless Bond number, which depends on the particle deposition geometry. I find particle rafts collapse when the sphere number $N=\alpha B o^{-2}+\beta B o^{-1}$, and particle stacks sink at the sphere number $N=\kappa B o^{-3 / 2}$. In the analogous microfluidic system, I control the size of the particles clusters by controlling the strength of the magnetic field gradient, and the interfacial tension of the ATPS liquid-liquid interface. I observe empirically that the number of particles within the cluster scales with the inverse of the product of a small aspect ratio and the dimensionless magnetic Bond number, $N=$ $\kappa^{\prime}\left(\varepsilon B o_{m}\right)^{-1}$.

I have developed a microfluidic self-assembly system with the motivation of using this technique for the conformal coating of cells and cell clusters. In my microfluidic system, I have demonstrated the self-assembly and coating of $10 \mu \mathrm{m}$ microparticles. Cells can be easily magnetized with a variety of functionalized magnetic microparticles or nanoparticles, and forced through the liquid-liquid interface in a similar manner. 
I have demonstrated the controlled self-assembly and coating of particle clusters, however polymerization of the coating layer is an essential component of this system which needs to be explored. For example, an ATPS consisting of photo-polymerizable polyethylene glycol diacrylate (PEGDA) and dextran could be used, where the clusters become coated in a layer of PEGDA which is UV polymerized downstream. Alternatively, an alginate coating layer could be formed, and polymerized via exposure to calcium chloride.

Although functionalized magnetic particles are an extremely convenient method of forcing cells through a liquid-liquid interface, residual cellular magnetism may not be desirable. Alternative forcing techniques could also be employed to pass the cells through the liquid-liquid interface, without the use of magnetism. For example, optical tweezers or acoustic waves could be used in place of a magnetic field gradient.

Collection of the coated clusters is also an open question. In this magnetic self-assembly system the clusters are collected in the PEG phase collection chamber, which is in the section of the cross-flow chamber which is closest to the magnet face, and thus where the magnitude of the magnetic force is strong in comparison to fluid shear forces. As a result, once the magnetic particle cluster reaches the collection area, it is difficult to remove them without also removing the magnetic field gradient. As a result, the above described coating system is not a continuous process, and at best would require two stages; first self-assembly and coating with the magnet field gradient applied, then collection once the magnetic field has been removed.

In addition to the variety of potential physical studies in this area, the long-term viability of cells which undergo this coating process must also be studied. While it is well understood that a thinner coating layer can improve the diffusion of molecules through the membrane, and thus the metabolic activity of the coated cells, the longterm integrity of the coating layer must be evaluated in a biologically active system. Despite these challenges, there have already been several promising studies on the viability of encapsulated cells $[16,52,66,67]$, and microfluidic technologies continue to provide opportunities to further improve the performance of this microencapsulation technique. 


\section{Appendix A}

\section{Microfluidic generation of aqueous two-phase system droplets by controlled pulsating inlet pressures}

\section{A.1 Abstract}

I present a technique that generates droplets using ultra-low interfacial tension aqueous two-phase systems. My method combines a classical microfluidic flow focusing geometry with precisely controlled pulsating inlet pressure, to form monodisperse ATPS droplets. The DEX disperse phase enters through the central inlet with variable on-off pressure cycles controlled by a pneumatic solenoid valve. The continuous phase PEG solution enters the flow focusing junction through the cross channels at a fixed flow rate. The on-off cycles of the applied pressure, combined with the fixed flow rate cross flow, make it possible for the ATPS jet to break up into droplets. I observe different droplet formation regimes with changes in the applied pressure magnitude and timing, and the continuous phase flow rate. I also develop a scaling model to predict the size of the generated droplets, and the experimental results show a good quantitative agreement with my scaling model. Additionally, I demonstrate the potential for scaling-up of the droplet production rate, with a simultaneous two-droplet generating geometry. I anticipate that this simple and precise approach to making ATPS droplets will find utility in biological applications where the all-biocompatibility of ATPS is desirable. 


\section{A.2 Introduction}

ATPS are formed by the mixture of incompatible polymer solutions, most commonly PEG and DEX $[68,69]$. Above a critical polymer concentration, the mixture separates into two distinct aqueous phases, the lower density PEG-rich phase and the higher density DEX-rich phase $[11,70]$. The phase separation of ATPS is excellent for applications in protein separation and extraction [71, 72], cell partitioning $[73,74,75]$, bacterial and cell micropatterning [76], and DNA extraction [77, 78]. As a result of their biocompatibility and selectivity, equilibrated ATPS phases are also useful for biological applications that require multiple fluid phases.

In microfluidics, oil-water two-phase systems have been widely used to produce discrete monodisperse droplets $[79,80,81]$. These systems typically employ hydrodynamically-controlled flow focusing geometries to generate droplets, by exploiting the Rayleigh-Plateau instability of a central liquid jet within a continuous outer phase fluid (either water-in-oil or oil-in-water). Due to the relatively high interfacial tension (typical oil-water systems have interfacial tension $\gamma=1-20 \mathrm{mN} \mathrm{m}^{-1}$ ), the liquid jet breaks up into small monodisperse droplets. The produced droplets vary in size from pico to nanoliters, and are used in reaction applications [79], particle synthesis [82], high-throughput assays [83], and single cell analysis [84]. However, to use these droplets in biological applications requires extensive post-processing due to the toxic nature of the oil phase [85].

ATPS was recently introduced into microfluidic devices, and already demonstrates the benefits of their biocompatibility, namely, in separating cells and proteins in a continuous manner [43, 86], in encapsulating cells [87], and in biomolecule delivery [75], However, due to the ultra-low interfacial tension of ATPS (typically $\gamma=0.1-100 \mu \mathrm{Nm}^{-1}$ ) $[12,13]$, most ATPS microfluidics experiments have been limited to the manipulation of simple laminar flows. The ultra-low interfacial tension of ATPS makes drop breakup in microchannels by the classical Rayleigh-Plateau instability difficult to achieve [88].

As a result, microfluidic ATPS droplet generation relies on application of external forcing. For example, microfluidic ATPS droplet generation is possible by electrohydrodynamic perturbation of the ATPS interface at a T-junction $[89,90]$. Squeezing an ATPS jet to cause its breakup into droplets is possible with mechanical forcing from an oscillating piezoelectric disk, embedded next to a microchannel 
[91, 92]. Lai et al. [93] also show monodisperse ATPS droplet formation with pin actuation in a rounded multi-level microchannel. Additionally, ATPS droplets and double emulsions can be achieved in glass capillary microfluidic devices, through controlled mechanical vibration of the flexible tubing that is connected to the inner stream of the capillary $[94,95,96]$.

Despite these approaches, generating ATPS droplets in microfluidics remains challenging. Namely, none of the existing methods are based on direct control of the flow into the microchannel. Consequently, there does not appear to be a simple way to modify the channel designs such that many monodisperse droplets are produced at once.

Here, I present a simple, hydrodynamically-controlled system, for ATPS droplet generation in polydimethylsiloxane (PDMS) based microfluidic channels. I modulate the hydrodynamics by combining fluid inlets of oscillating pressure and constant flow rate. The disperse phase is injected into the inner channel with a pulsating applied pressure that is controlled by a solenoid valve, and the continuous phase enters the cross-flow inlet via a constant flow rate syringe pump. I experimentally obtain different drop formation regimes, and tune the resulting droplet size as I vary the magnitude and the 'on' and 'off' times of the applied pressure, and the continuous phase flow rate. I also develop a simple scaling model to predict the ATPS droplet sizes, and I find a good agreement with the experimental results. Finally, I demonstrate the potential scale-up of my ATPS droplet generation approach, by simultaneously forming double droplets with a single pressure source in a single microchannel.

The uniqueness of my approach lies in its simplicity: I use a conventional flow-focusing PDMS-glass microfluidic device without any additional components, and the square-wave pulsating pressure that I apply can be easily replicated by a commercially available pressure-controlled syringe pump (for example, the Fluigent MFCS-EZ). I anticipate that this simple technique will help to democratize microfluidic generation of monodisperse ATPS droplets. 


\section{A.3 Experimental methods}

\section{A.3.1 Chemicals}

I phase-separate an aqueous solution containing $10 \%$ (w/v) polyethylene glycol (PEG, Mw 8,000, SigmaAldrich, St. Louis, MI, USA) and 5 \% (w/v) dextran (DEX, Mw 100,000, Sigma-Aldrich, St. Louis, MI, USA) for 24 hours, into the upper PEG-rich phase and the lower DEX-rich phase. Once equilibrated, I use syringes to isolate the two phases. I use a glass viscometer to measure the PEG-rich phase and DEX-rich phase viscosities $\mu_{P}=8.7 \mathrm{mPa}$ s and $\mu_{D}=99.8 \mathrm{mPa}$ s, respectively, and I use reports in the literature to estimate their ultra-low interfacial tension $\gamma=O(10-100) \mu \mathrm{N} \mathrm{m}^{-1}[97,98]$.

\section{A.3.2 Device fabrication}

The microfluidic device is fabricated using standard soft lithography methods [60]. I draw the flow focusing channel geometry with computer-aided design (CAD) software (AutoCAD 2010, Autodesk, Inc., Dan Rafael, CA, USA) and print onto a transparency sheet (25,400 dpi, CAD/ART Services Inc., Bandon, OR, USA) to make the photomask. I spin-coat SU-8 2035 photoresist (Microchem., Newton, MA USA) onto a 4 inch silicon wafer (University Water Inc., Boston, MA, USA) and then expose the wafer to a UV light through the transparency photomask. After chemical development, the desired microchannel pattern is formed on the silicon master.

To make PDMS-glass bonded channels, I pour a 10:1 ratio mixture of PDMS resin and curing agent (Sylgard 184, Dow-Corning, Midland, MI, USA) onto the prepared silicon master, and cure it in an oven for 2 hours. A biopsy punch (Integra Miltex, Inc., Rietheim-Weilheim, Germany) is used to create inlet and outlet holes in the PDMS layer. I clean the PDMS sheet and a cover glass slide (Corning microscope slides $75 \times 25 \mathrm{~mm}$, Sigma-Aldrich, St. Louis, MI, USA) by sonication in ethanol, and dry both with nitrogen gas. After oxygen plasma treatment (Harrick Plasma, Ithaca, NY, USA), I bond the PDMS layer and glass slide irreversibly. 


\section{A.3.3 Experiment setup}

ATPS droplets are generated by incorporating pulsating applied pressure and constant flow rates at the system inlets (Fig. A.1 (a)). I load the DEX solution into a $250 \mu \mathrm{L}$ pipette tip, and vertically insert the tip into the inner stream inlet of the microfluidic device. A Tygon tubing (Saint-Gobian, La Défense, Courbevoie, France) is interfaced with the other end of the pipette tip, and is used to transport compressed air to the DEX solution, to inject the solution into the microchannel.

In Fig. A.1 (b), the DEX channel and downstream channel widths, $w=50 \mu \mathrm{m}$ and $w_{c}=150 \mu \mathrm{m}$, respectively. The DEX channel length $\ell=5 \mathrm{~mm}$, from the DEX inlet to the flow focusing junction. All microchannels have height, $h=50 \mu \mathrm{m}$.

A pressure regulator (Type 100LR, Control Air Inc., Amherst, NH, USA) is serially connected to a three-way solenoid valve (Model 6014, Burkert, Germany), to continuously tune the on-off cycles of the compressed air. The solenoid valve has a response time $10-20 \mathrm{~ms}$. This setup is similar to experimental systems used in stop-flow lithography [99]. I control the valve by a customized LabVIEW (National Instruments, Austin, Texas, USA) program, and apply 'on' DEX inlet pressures $P_{o}=21$ or $42 \mathrm{kPa}$. The PEG cross-flow is supplied via a constant flow rate syringe pump at flow rates $Q_{o}=1$ or $3 \mu \mathrm{L} \mathrm{min}{ }^{-1}$ (Harvard Apparatus, Holliston, MA, USA).

Experimental images of ATPS droplets are captured using an inverted microscope (Axio Observer.A1, Zeiss, Oberkochen, Germany) with an attached high speed camera (Vision Research, Wayne, NJ, USA). The high speed camera operates at a frame rate of $500 \mathrm{fps}$ and an exposure time of $1000 \mathrm{\mu s.}$ I use ImageJ software to post-process the images and measure the size of droplets.

\section{A.4 Results and discussion}

\section{A.4.1 Making ATPS droplets}

I produce ATPS droplets in a flow focusing geometry, by application of a pulsating applied pressure to the disperse phase inlet, and a constant flow rate to the continuous phase inlet. Fig. A.1 (b) shows that the disperse DEX solution pressure $P$ switches between 'on', $P=P_{o}$, and 'off', $P=0$. The corresponding 


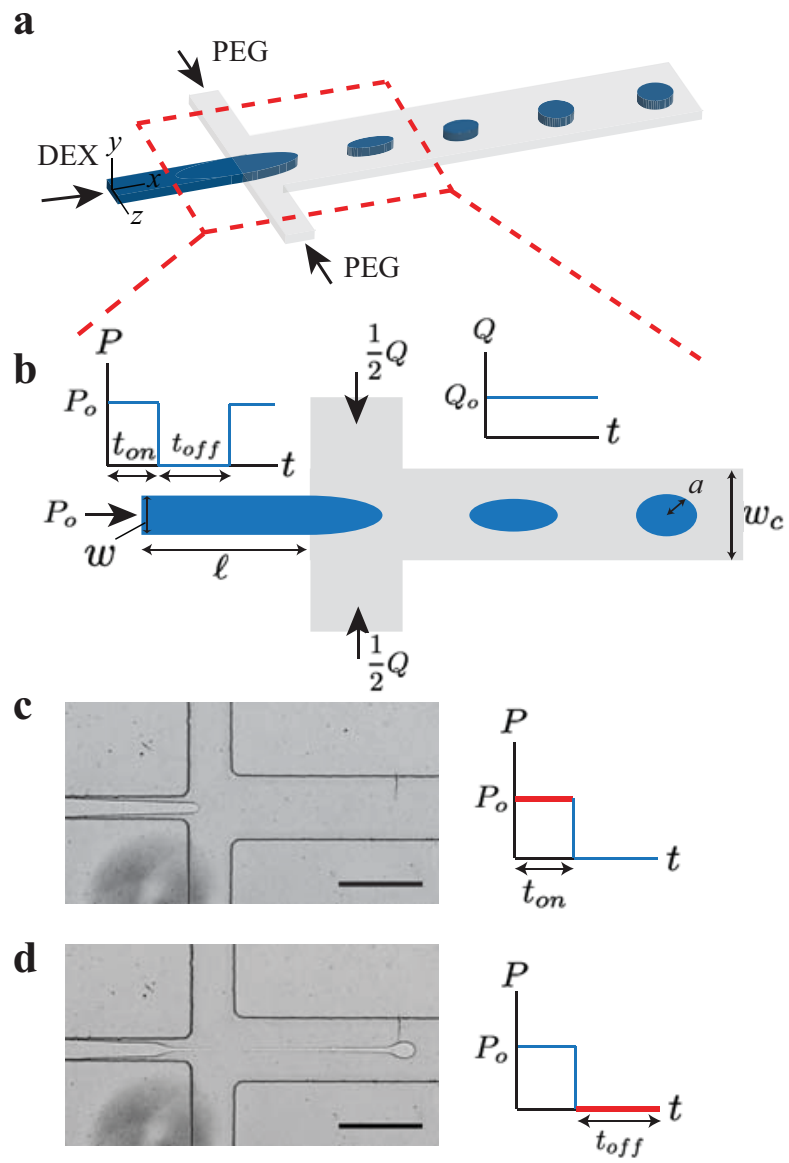

Figure A.1: (a) Schematic diagram of ATPS droplet formation in a flow-focusing geometry. The DEX solution is injected by an applied pressure and the PEG solution is introduced by a syringe pump. (b) Droplet formation mechanism. A solenoid valve controls the on-off cycle of the applied pressure of the DEX inlet, and the PEG is introduced at a constant flow rate. When the inlet pressure is applied, (c) the pressure pushes the DEX solution into the cross-junction. With the inlet pressure off, (d) the DEX solution breaks into drops by the constant flow rate of the PEG sheath flow. Scale bar $200 \mu \mathrm{m}$. 
'on' and 'off' times are $t_{o n}$ and $t_{o f f}$, respectively. The continuous phase PEG is introduced at a constant flow rate, $Q=Q_{o}$, and enters via a single inlet which subsequently splits into the two sides of the cross flow at the flow focusing junction (Fig. A.1 (b)).

During the pressure on-time $t_{\text {on }}$, the applied pressure pushes the DEX phase into the junction (Fig. A.1 (c)). Then when the applied pressure is 'off', the constant flow rate of the continuous PEG phase shears a droplet off the DEX jet (Fig. A.1 (d)). This continued on-off modulation of the applied pressure to the DEX phase, coupled with a constant flow rate of the outer PEG phase, enables generation of a steady stream of monodisperse DEX droplets.

Such ATPS droplet formation would not be possible with conventional droplet-making microfluidic flow focusing systems that apply constant flow rates for the disperse and continuous phases. Using a conventional setup with an ultra-low interfacial tension ATPS, would result in a long and steady jet of the disperse phase, which, in the absence of external forcing, will not break into droplets within the length of the microchannel.

\section{A.4.2 Different regimes of ATPS interfacial dynamics}

To investigate the behavior of the ATPS interface at the flow focusing junction, I fix the DEX 'on' pressure $P_{o}$ and the PEG constant flow rate $Q_{o}$, and sweep across a range of DEX pressure 'on' and 'off' times, $t_{o n}$ and $t_{o f f}$, respectively. Fig. A.2 (a) shows the phase diagram I obtain for the ATPS interfacial dynamics at the junction of the microchannel system. Here, the DEX 'on' pressure $P_{o}=21 \mathrm{kPa}$, and the PEG outer flow rate $Q_{o}=1 \mu \mathrm{L} \mathrm{min}^{-1}$. I observe that the range of DEX pressure off-time $t_{o f f}$, that permits droplet generation (solid and empty diamonds), grows with increasing DEX pressure on-time $t_{\text {on }}$.

As shown in Fig. A.2 (a) and (b), a small DEX pressure 'off' time, $t_{\text {off }}<100$ ms, results in wide DEX jets that have a large wavelength interfacial perturbation (see top-left image in Fig. A.2 (b)). This deformation of the interface decreases downstream as the DEX-PEG interface stabilizes. A similar behavior occurs at a higher DEX pressure 'off' time, $100<t_{o f f}<300 \mathrm{~ms}$, where the DEX jet is more narrow and attains a similar interfacial perturbation (see middle-left image in Fig. A.2 (b)). In these two regimes, the shear stress from the PEG sheath flow is insufficient to completely break up the DEX 

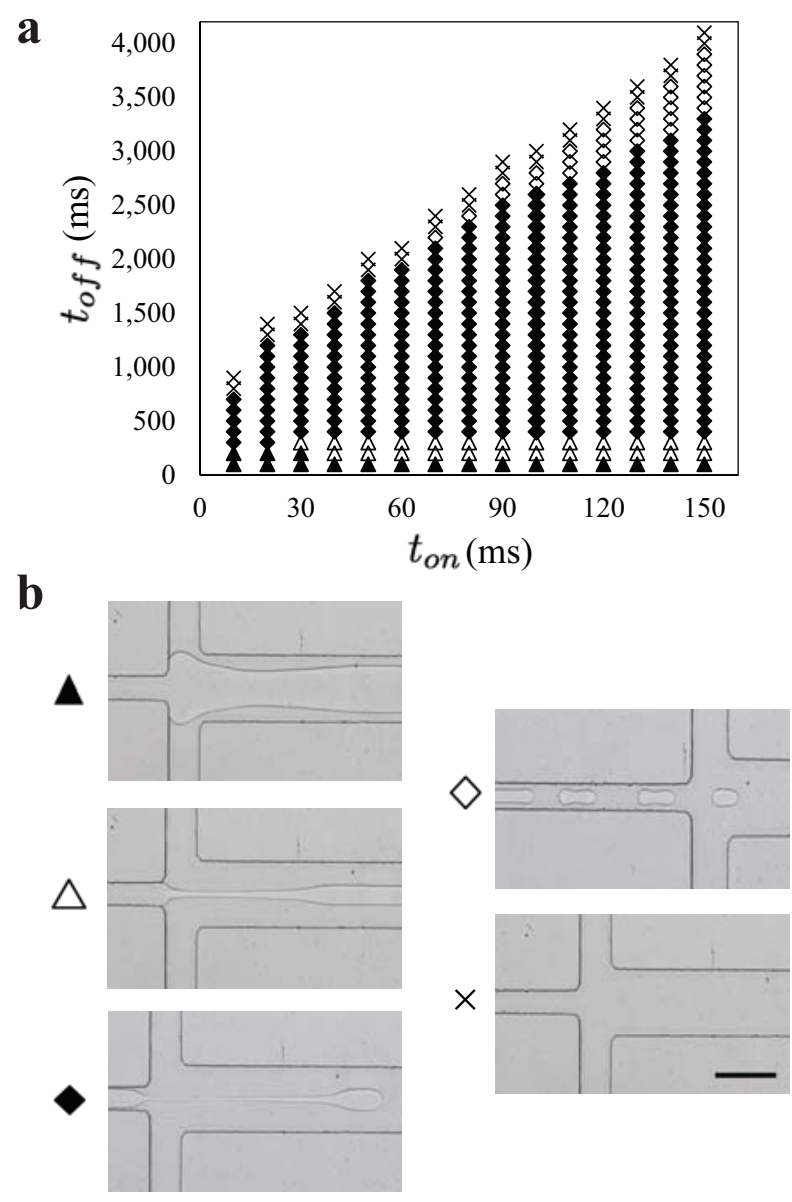

Figure A.2: (a) Phase diagram of ATPS interfacial dynamics in my microchannel. For this phase diagram, the inner DEX stream 'on' pressure $P_{o}=21 \mathrm{kPa}$ and the outer PEG flow rate $Q_{o}=1 \mu \mathrm{L}$ $\mathrm{min}^{-1}$. I sweep across a range of pressure-on and pressure-off times, $t_{\text {on }}$ and $t_{\text {off }}$, respectively, to investigate the resulting dynamics. Solid and empty diamonds correspond to regimes that allow for ATPS droplet formation. (b) Representative images of flow patterns observed in the microchannel: wide jets (solid triangles), narrow jets (empty triangles), downstream droplet formation (solid diamonds), upstream droplet formation (empty diamonds), and DEX phase backflow (crosses). Scale bar $200 \mu \mathrm{m}$. 
jet into droplets.

Further increase of DEX pressure off-time, $t_{\text {off }}>300 \mathrm{~ms}$, causes the PEG sheath flow to push the DEX jet into a neck (solid diamonds in Fig. A.2 (a) and (b)). Eventually, the neck ruptures and a drop is formed upstream of the neck. This droplet generation regime is observed throughout a wide range of DEX pressure on-time and off-time values, $t_{o n}$ and $t_{o f f}$, respectively. I note that in this regime, trailing secondary drops are observed, and these drops follow the main droplet along the downstream channel.

Interestingly, I find a second droplet generation regime (empty diamonds) that is visually distinct from the previous regime (solid diamonds). Namely, at higher values of DEX pressure off-time $t_{o f f}$, I observe that small droplets are formed in the DEX channel that is upstream of the flow focusing junction (top-right image in Fig. A.2 (b)). In this regime, the extended period of zero pressure in the DEX inlet causes a backflow of the disperse DEX phase and the continuous PEG phase into the upstream channel. The pull-back of the DEX jet also results in the formation of a neck in the upstream channel, which subsequently ruptures to form a DEX drop. The DEX droplet gets pushed into the flow focusing junction when the DEX inlet pressure is finally 'on'.

At even higher values of pressure 'off' time $t_{o f f}$, none of the DEX phase enters the cross-junction. Therefore, no droplets are generated in this regime (crosses in Fig A.2 (a) and bottom-right image of Fig. A.2 (b)).

I also study how the droplet formation regimes change with the value of the DEX phase applied pressure $P_{o}$, and the PEG phase flow rate $Q_{o}$. Fig. A.3 shows a phase diagram with four DEX and PEG inlet combinations. Namely, the DEX phase 'on' pressure $P_{o}=21$ or $42 \mathrm{kPa}$, and the PEG phase flow rate $Q_{o}=1$ or $3 \mu \mathrm{L} \min ^{-1}$.

Across a range of pressure 'on' and 'off' times, $t_{o n}$ and $t_{o f f}$, respectively, I find that the drop-making regime expands with increasing disperse phase applied pressure $P_{o}$ and decreasing continuous phase flow rate $Q_{o}$. This result is mainly due to the backflow of the DEX phase at higher values of the DEX pressure 'off' time $t_{\text {off }}$. At higher DEX phase applied pressure $P_{o}$, the pressure 'off' time $t_{\text {off }}$ can be longer without causing DEX phase backflow into the upstream channel. Lowering the continuous PEG phase flow rate $Q_{o}$ also helps to prevent backflow of the DEX phase. Therefore, the combination that achieves the largest droplet formation range is at a high DEX applied pressure $P_{o}=42 \mathrm{kPa}$ and a low 


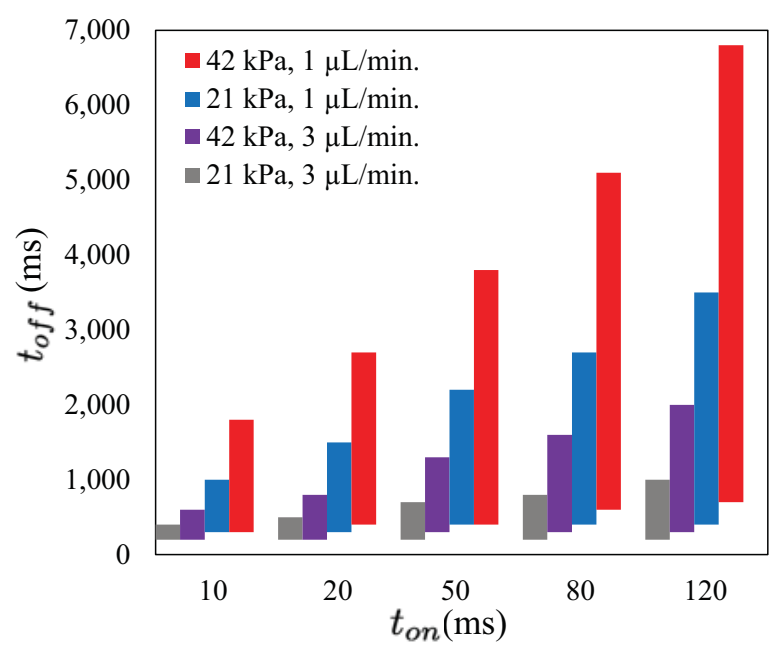

Figure A.3: Phase diagram of regimes that permit droplet generation. Here, I apply DEX 'on' pressures $P_{o}=21$ or $42 \mathrm{kPa}$ and PEG flow rates $Q_{o}=1$ or $3 \mu \mathrm{L} \mathrm{min}{ }^{-1}$, and sweep across a range of DEX pressure 'on' and 'off' times, $t_{o n}$ and $t_{\text {off }}$.

PEG flow rate $Q_{o}=1 \mu \mathrm{L} \mathrm{min}^{-1}$.

\section{A.4.3 Elongated ATPS droplet shapes}

In my system, the ultra-low ATPS interfacial tension $\left(\gamma=O(10) \mu \mathrm{N} \mathrm{m}^{-1}\right)$ results in a large capillary number $C a=\mu_{P} U / \gamma>1$, provided that the DEX applied pressure is 'on' and produces an average flow speed $U$. As a result of the dominance of shear stress over interfacial tension, I observe that as the DEX droplets move downstream in the channel, they form an elongated shape (Fig. A.4 (a)). This effect is more pronounced in larger DEX droplets, and may be exploited in the future to, for example, quantify the ultra-low interfacial tension of the ATPS solution, by measuring the aspect ratio of the elongated droplets.

Unlike in conventional oil-water systems, in my ultra-low interfacial tension system, the shear stress from the fluid flow is able to overcome the tendency of drops to minimize their interfacial area and form spheres. A conventional oil-water emulsion would tend to expand to the sidewalls of the microchannel or form spheres in the microchannel $[100,101,102]$. Here, as the drops flow into outlet reservoir, they are no longer under shear stress from the flow, so the drops become spheres (Fig. A.4 (b)). 
$\mathbf{a}$
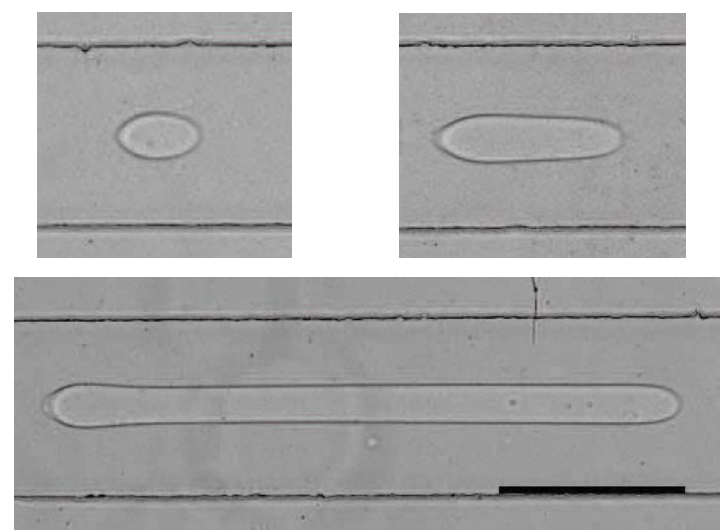

b

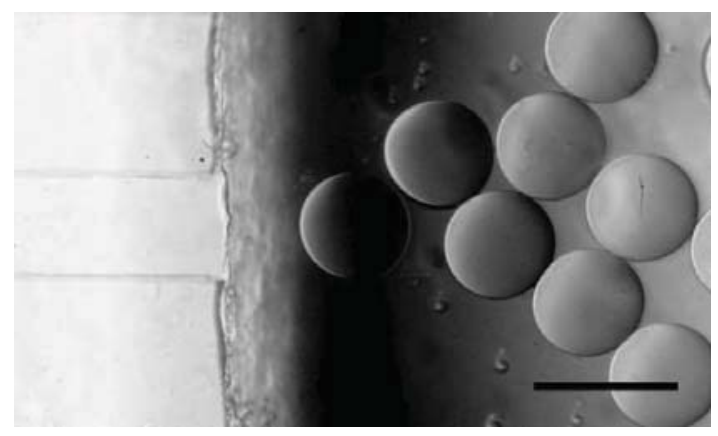

Figure A.4: (a) Different size and shape of droplets downstream in the microchannel. Due to the high capillary number in the flow, the droplets have elongated shapes. (b) The droplets become spheres as they move into the outlet reservoir, where the flow slows dramatically and the drops are no longer confined in the channel. Scale bar $200 \mu \mathrm{m}$. 


\section{A.4.4 Controlling the size ATPS droplets}

Figs. A.5 (a) and (b) show values of the resulting DEX droplet radius $a$, plotted against the DEX phase inlet pressure 'on' time $t_{o n}$ (Fig. A.5 (a)) and 'off' time $t_{\text {off }}$ (Fig. A.5 (b)). In Fig. A.5 (a) the 'off' time $t_{\text {off }}=600 \mathrm{~ms}$, and in Fig. A.5 (b) the 'on' time $t_{o n}=80 \mathrm{~ms}$. Here, I use four combinations of the DEX phase 'on' pressure $P_{o}=21$ or $42 \mathrm{kPa}$, and PEG phase constant flow rate $Q_{o}=1$ or $3 \mu \mathrm{L} \mathrm{min}{ }^{-1}$, to obtain the experimental results, and I measure the radius of the drops at the microchannel reservoir (Fig. A.4 (b)).

The plots (Figs. A.5 (a) and (b)) show monotonic increasing droplet radius $a$ with longer DEX phase pressure 'on' times $t_{o n}$, and shorter 'off' times $t_{o f f}$. I also note that DEX phase drop radius a grows with higher DEX phase applied pressure $P_{o}$, and with lower PEG phase flow rate $Q_{o}$. The droplets that I produce range in size from $a=22$ to $177 \mu \mathrm{m}$.

In the limit of higher values of the DEX phase pressure 'on' time (such that the transient ramp-up time of the flow speed can be neglected), I note that the droplet volume is proportional to the product of the resulting DEX phase flow rate $Q_{D}$ and the 'on' time $t_{\text {on }}$, and inversely proportional to the PEG phase flow rate $Q_{o}$ and the DEX phase pressure 'off' time $t_{o f f}$,

$$
\left(\frac{a}{w}\right)^{3} \propto \frac{Q_{D}}{Q_{o}} \frac{t_{o n}}{t_{o f f}}
$$

where, during the time when the DEX phase pressure is applied, the steady-state DEX phase flow rate $Q_{D}=U w h$. Here, $U$ is the average speed of the DEX phase. The Hele-Shaw type Stokes flow relationship in the flow $(x)$ direction,

$$
\frac{\partial P}{\partial x} \approx \mu_{D} \frac{\partial^{2} u}{\partial y^{2}}
$$



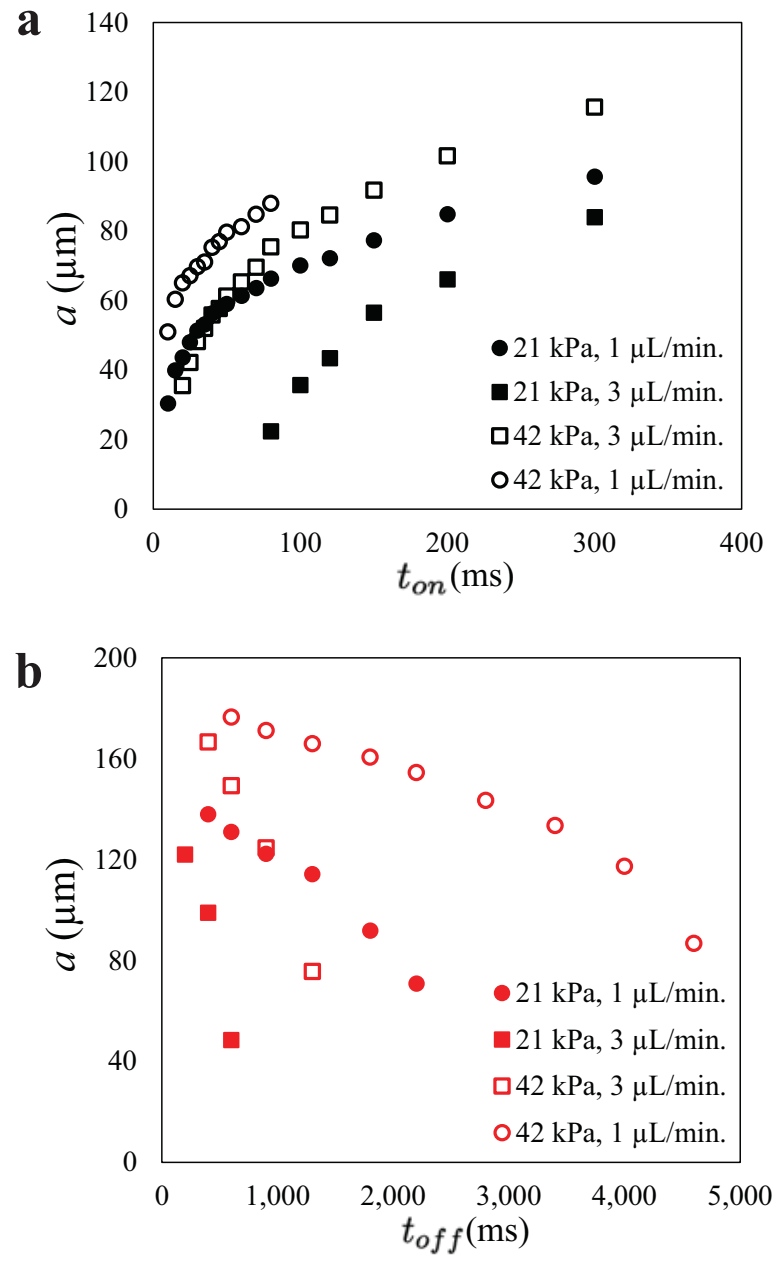

Figure A.5: Plots of measured DEX droplet radius $a$ versus the 'on' and 'off' times of the DEX phase inlet pressure. I plot four different combinations of experimental conditions with the applied DEX pressure $P_{o}=21$ or $42 \mathrm{kPa}$, and PEG flow rate $Q_{o}=1$ or $3 \mu \mathrm{L} \mathrm{min}{ }^{-1}$. (a) Drop radius $a$ versus DEX pressure 'on' time $t_{o n}$, while the 'off' time $t_{o f f}=600 \mathrm{~ms}$. (b) Drop radius $a$ versus the DEX phase pressure 'off' time $t_{\text {off }}$, while the 'on' time $t_{\text {on }}=80 \mathrm{~ms}$. 
where $u$ is the flow velocity, and $y$ is the direction normal to the flow along the channel height (Fig. A.1 (a)). Eqn. (A.2) gives the scaling representation of the average flow speed $U$,

$$
U \propto \frac{h^{2}}{\ell} \frac{P_{o}}{\mu_{D}}
$$

Substituting $Q_{D}=U w h$ and Eqn. (A.3) into Eqn. (A.1), I obtain the scaling relationship for the generated DEX droplet radius,

$$
\frac{a}{w}=\kappa\left(\frac{w h^{3}}{\ell} \frac{P_{o} t_{o n}}{\mu_{D} Q_{o} t_{o f f}}\right)^{1 / 3}
$$

which has the proportionality constant $\kappa$.

Eqn. (A.4) gives the prediction that DEX droplets will be larger with higher values of the applied pressure, $P_{o}$, and the time $t_{o n}$ over which the pressure is 'on'. The model also indicates that the radius of the droplets will decrease with increasing continuous PEG phase flow rate, $Q_{o}$, and the DEX phase pressure 'off' time, $t_{\text {off }}$. All of this is qualitatively consistent with my experimental observations (see for example Figs. A.5 (a) and (b)).

Fig. A.6 is a log-log plot of the dimensionless DEX droplet radius $a / w$ versus the dimensionless parameter $w h^{3} P_{o} t_{o n} / \ell \mu_{D} Q_{o} t_{o f f}$. All of the experimental results from Figs. A.5 (a) and (b) collapses onto a single curve, and display a good quantitative agreement with with my scaling model in Eqn. (A.4). Namely, the experimental data reflect my predicted $1 / 3$ power-law, and I obtain an empirical value of the proportionality constant $\kappa \approx 0.53$, by fitting my scaling model with the experimental data. The agreement between my model and experiments indicates the utility of the model (Eqn. (A.4)) for designing future microfluidic ATPS emulsion generation schemes. 


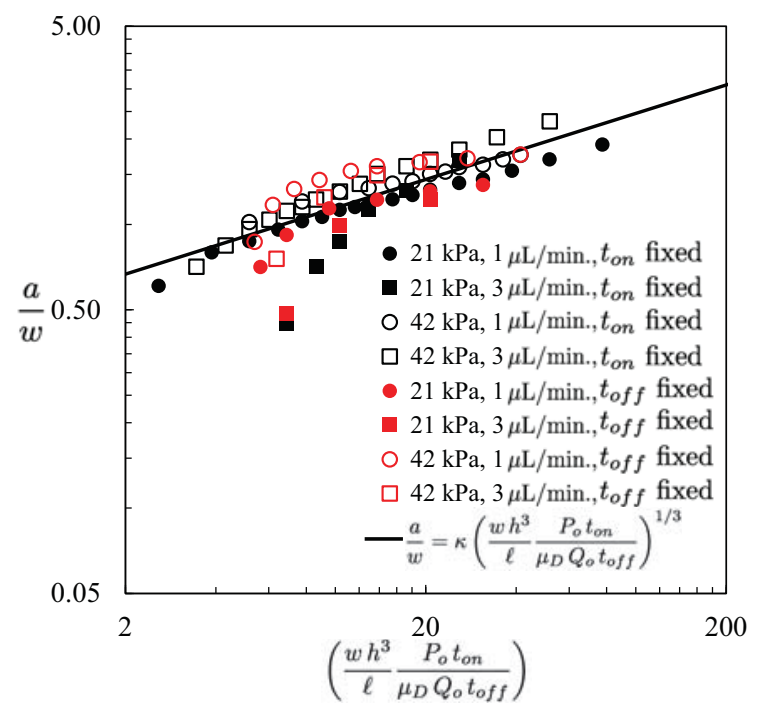

Figure A.6: $\quad$ Log-log plot of the ATPS droplet dimensionless radius $a / w$, versus the dimensionless parameter $w h^{3} P_{o} t_{o n} / \ell \mu_{D} Q_{o} t_{o f f}$. Experimental values from eight different combinations of flow conditions are shown, and the results show a good agreement with the scaling model (solid line).

\section{A.4.5 Scaling-up to simultaneous double droplet formation}

One limitation of my ATPS droplet generation technique is that the production rate is typically $O(0.1)-$ $O(1)$ droplets per second. This limitation arises because the DEX phase applied pressure 'off' time $t_{\text {off }}$ has to be sufficiently long to enable DEX droplets to form (Fig. A.2 (a)). However, I may be able to overcome this limitation by scaling-up my droplet production process.

Fig. A.7 shows a proof-of-concept scale-up of my microfluidic ATPS droplet generation technique. Here, I take advantage of a parallel flow microfluidic setup [103, 104, 105], to design a multiplexed ATPS droplet generator. I apply a single pressure source, $P_{o}$ at the DEX phase inlet. With a branched inlet channel design, the DEX phase flows into the flow focusing junction from two channels. The continuous phase PEG flows at a constant flow rate $Q_{o}$, as before. $5 \%(\mathrm{w} / \mathrm{v})$ DEX and $40 \%(\mathrm{w} / \mathrm{v})$ PEG are used for this experiment. The DEX phase $(5 \%(\mathrm{w} / \mathrm{v}))$ is supplied with an 'on' pressure $P_{o}=21 \mathrm{kPa}$. Pressure 'on' and 'off' times $t_{o n}=70 \mathrm{~ms}$ and $t_{o f f}=600 \mathrm{~ms}$, respectively.

The image in Fig. A.7 shows the proof-of-concept of the scaling-up of my method. Since multiplexing of a pressure-driven flow is possible with a parallel flow system, my pressure-assisted ATPS emulsion 


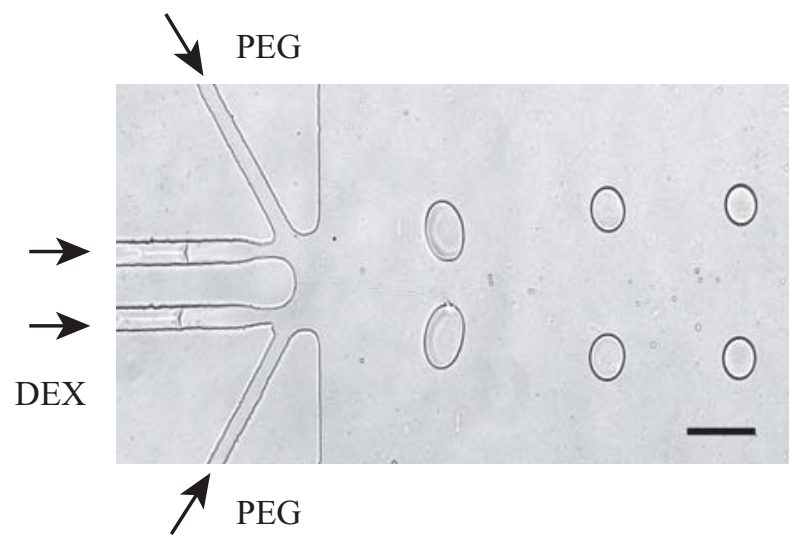

Figure A.7: Image of simultaneous multiple ATPS droplet formation in the microchannel. Two drops are generated at the same time with a single pressure source. Here, the continuous PEG solution (40 $\%(\mathrm{w} / \mathrm{v}))$ is flowed at $1 \mu \mathrm{L} \mathrm{min}{ }^{-1}$, and the disperse DEX phase $(5 \%(\mathrm{w} / \mathrm{v}))$ is injected with an 'on' pressure $P_{o}=21 \mathrm{kPa}$. Pressure 'on' and 'off' times $t_{o n}=70 \mathrm{~ms}$ and $t_{o f f}=600 \mathrm{~ms}$, respectively. Scale bar $200 \mu \mathrm{m}$.

making technique has the potential to scale-up massively via many parallel disperse phase channels. To demonstrate this approach, a parallel integrated ATPS droplet generator is now under investigation in my group, to more significantly increase the production rate of ATPS droplets.

\section{A.5 Conclusions}

I demonstrate a microfluidic ATPS droplet formation system that utilizes a pulsating applied pressure, and hydrodynamic flow focusing. The on-off pressure cycles of the disperse DEX phase, in combination with the constant flow rate continuous PEG phase, make it possible to controllably produce monodisperse ATPS droplets in a flow focusing junction.

I experimentally observe different droplet formation regimes that depend on experimental parameters such as the applied pressure magnitude $P_{o}$, the continuous phase flow rate $Q_{o}$, and the 'on' and 'off' times of the applied pressure $t_{o n}$ and $t_{\text {off }}$, respectively. I also produce droplets that vary by an order of magnitude in volume, from $O(10) \mathrm{pL}$ to $O(10) \mathrm{nL}$, and my simple scaling model captures quantitatively the physics that determine the resulting droplet size. Finally, I show the proof-of-concept of the scalingup of my ATPS droplet making scheme, by multiplexing the formation of droplets in a parallel-flow 
system.

Our microfluidic platform offers a simple method to create monodisperse ATPS droplets. This setup overcomes the challenges associated with the ultra-low interfacial tension of ATPS, by combining a pulsating disperse phase pressure, and a constant continuous phase flow rate. This method is applicable to on-demand drop formation applications $[106,107]$. I also anticipate that this approach may have utility in biological applications where the biocompatible nature of ATPS is desirable, for example, in cell encapsulation [16], particle coating [9, 10], and drug delivery [108]. 



\section{Bibliography}

[1] H. Li, J. D. Carter, and T. H. LaBean. Nanofabrication by DNA self-assembly. Mater. Today, 12(5):24-32, 2009.

[2] N. A. Kotov, I. Dekany, and J. H. Fendler. Layer-by-layer self-assembly of polyelectrolytesemiconductor nanoparticle composite films. J. Phys. Chem. A, 99(35):13065-13069, 1995.

[3] J. N. Israelachvili, D. J. Mitchell, and B. W. Ninham. Theory of self-assembly of hydrocarbon amphiphiles into micelles and bilayers. J. Chem. Soc., Faraday Trans. 2, 72:1525-1568, 1976.

[4] D. E. Discher and Adi. Eisenberg. Polymer vesicles. Science, 297(5583):967-973, 2002.

[5] G. A. Ozin, K. Hou, B. V. Lotsch, L. Cademartiri, D. P. Puzzo, F. Scotognella, A. Ghadimi, and J. Thomson. Nanofabrication by self-assembly. Mater. Today, 12(5):12-23, 2009.

[6] G. M. Whitesides and B. Grzybowski. Self-assembly at all scales. Science, 295(5564):2418-2421, 2002.

[7] D. Vella and L. Mahadevan. The cheerios effect. Am. J. Phys., 73(9):817-825, 2005.

[8] N. Vandewalle, N. Obara, and G. Lumay. Mesoscale structures from magnetocapillary selfassembly, 2013.

[9] S. S. H. Tsai, J. S. Wexler, J. Wan, and H. A. Stone. Conformal coating of particles in microchannels by magnetic forcing. Appl. Phys. Lett., 99(15):153509, 2011.

[10] B-U. Moon, N. Hakimi, D. K. Hwang, and S. S. H. Tsai. Microfluidic conformal coating of nonspherical magnetic particles. Biomicrofluidics, 8(5):052103, 2014.

[11] P. Albertsson. Partition of cell particles and macromolecules: separation and purification of biomolecules, cell organelles, membranes, and cells in aqueous polymer two-phase systems and their use in biochemical analysis and biotechnology, volume 346. Wiley New York etc., 1986.

[12] R. Hatti-Kaul. Aqueous two-phase systems. Mol. Biotechnol., 19(3):269-277, 2001.

[13] E. Atefi, J. A. Mann Jr, and H. Tavana. Ultralow interfacial tensions of aqueous two-phase systems measured using drop shape. Langmuir, 30(32):9691-9699, 2014. 
[14] J. Ryden and P. Albertsson. Interfacial tension of dextran-polyethylene glycol-water two-phase systems. J. Colloid Interface Sci., 37(1):219-222, 1971.

[15] S. S. H. Tsai, I. M. Griffiths, Z. Li, P. Kim, and H. A. Stone. Interfacial deflection and jetting of a paramagnetic particle-laden fluid: theory and experiment. Soft Matter, 9(35):8600-8608, 2013.

[16] G. Orive, R. M. Hernández, A. R. Gascón, R. Calafiore, T. M. S. Chang, P. De Vos, G. Hortelano, D. Hunkeler, I. Lacík, A. M. J. Shapiro, and J. L. Pedraz. Cell encapsulation: promise and progress. Nat. Med, 9(1):104-107, 2003.

[17] J. A. Giraldo, J. D. Weaver, and C. L. Stabler. Enhancing clinical islet transplantation through tissue engineeering strategies. J. Diabetes Sci. Technol., 4(5):1238-1247, 2010.

[18] V. L. Workman, S. B. Dunnett, P. Kille, and D. D. Palmer. Microfluidic chip-based synthesis of alginate microspheres for encapsulation of immortalized human cells. Biomicrofluidics, 1(1):014105, 2007.

[19] Y-C. Tan, K. Hettiarachchi, M. Siu, Y-R. Pan, and A. P. Lee. Controlled microfluidic encapsulation of cells, proteins, and microbeads in lipid vesicles. J. Amer. Chem. Soc., 128(17):5656-5658, 2006.

[20] J. Clausell-Tormos, D. Lieber, J-C. Baret, A. El-Harrak, O. J. Miller, L. Frenz, J. Blouwolff, K. J. Humphry, S. Köster, H. Duan, C. Holtze, D. A. Weitz, A. D. Griffiths, and C. A. Merten. Droplet-based microfluidic platforms for the encapsulation and screening of mammalian cells and multicellular organisms. Chem. Biol., 15(5):427-437, 2008.

[21] D. Vella. Floating versus sinking. Annu. Rev. Fluid Mech., 47(1), 2014.

[22] J. W. M. Bush, D. L. Hu, and M. Prakash. The integument of water-walking arthropods: form and function. Adv. Insect Physiol., 34:117-192, 2007.

[23] X. Gao and L. Jiang. Biophysics: water-repellent legs of water striders. Nature, 432(7013):36-36, 2004.

[24] N. J. Mlot, C. A. Tovey, and D. L. Hu. Fire ants self-assemble into waterproof rafts to survive floods. Proc. Natl. Acad. Sci. U.S.A., 108(19):7669-7673, 2011.

[25] P. C. Foster, N. J. Mlot, A. Lin, and D. L. Hu. Fire ants actively control spacing and orientation within self-assemblages. J. Exp. Biol., 217(12):2089-2100, 2014.

[26] L. Wu, Z. Lian, G. Yang, and M. Ceccarelli. Water dancer II-a: a non-tethered telecontrollable water strider robot. Int. J. Adv. Robotic Sy., 8(4):10-17, 2011.

[27] O. Ozcan, H. Wang, J. D. Taylor, and M. Sitti. Surface tension driven water strider robot using circular footpads. In IEEE Int. Conf. Robot., pages 3799-3804. IEEE, 2010.

[28] Y. S. Song and M. Sitti. Surface-tension-driven biologically inspired water strider robots: Theory and experiments. IEEE Trans. Robot., 23(3):578-589, 2007. 
[29] M. Abkarian, S. Protière, J. M. Aristoff, and H. A. Stone. Gravity-induced encapsulation of liquids by destabilization of granular rafts. Nat. Commun., 4:1895, 2013.

[30] J. W. Tavacoli, G. Katgert, E. G. Kim, M. E. Cates, and P. S. Clegg. Size limit for particlestabilized emulsion droplets under gravity. Phys. Rev. Lett., 108(26):268306, 2012.

[31] J. W. Tavacoli, J. H. J. Thijssen, and P. S. Clegg. Particle-stabilized oscillating diver: a selfassembled responsive capsule. Soft Matter, 7(18):7969-7972, 2011.

[32] D. Vella, D-G Lee, and H-Y Kim. The load supported by small floating objects. Langmuir, 22(14):5979-5981, 2006.

[33] D. Vella, P. D. Metcalfe, and R. J. Whittaker. Equilibrium conditions for the floating of multiple interfacial objects. J. Fluid Mech., 549:215-224, 2006.

[34] J-L Liu, X-Q Feng, and G-F Wang. Buoyant force and sinking conditions of a hydrophobic thin rod floating on water. Phys. Rev. E, 76(6):066103, 2007.

[35] S Fordham. On the calculation of surface tension from measurements of pendant drops. In Proceedings of the Royal Society of London A: Mathematical, Physical and Engineering Sciences, volume 194, pages 1-16. The Royal Society, 1948.

[36] E. H. Mansfield, H. R. Sepangi, and E. A. Eastwood. Equilibrium and mutual attraction or repulsion of objects supported by surface tension. Phil. Trans. R. Soc. A, 355(1726):869-919, 1997.

[37] P. A. Kralchevsky and K. Nagayama. Capillary interactions between particles bound to interfaces, liquid films and biomembranes. Adv. Colloid Interface Sci., 85(2):145-192, 2000.

[38] G. Meng, N. Arkus, M. P. Brenner, and V. N. Manoharan. The free-energy landscape of clusters of attractive hard spheres. Science, 327(5965):560-563, 2010.

[39] V. N. Manoharan, M. T. Elsesser, and David J. Pine. Dense packing and symmetry in small clusters of microspheres. Science, 301(5632):483-487, 2003.

[40] J. B. Keller. Surface tension force on a partly submerged body. Phys. Fluids, 10(11):3009-3010, 1998.

[41] C-X. Zhao. Multiphase flow microfluidics for the production of single or multiple emulsions for drug delivery. Adv. Drug Deliv. Rev., 65(11):1420-1446, 2013.

[42] M. D. Tarn, M. J. Lopez-Martinez, and N. Pamme. On-chip processing of particles and cells via multilaminar flow streams. Anal. Bioanal. Chem., 406(1):139-161, 2014.

[43] J. R. SooHoo and G. M. Walker. Microfluidic aqueous two-phase system for leukocyte concentration from whole blood. Biomed. Microdevices, 11(2):323-329, 2009. 
[44] A. Sinha, A. K. Mollah, S. Hardt, and R. Ganguly. Particle dynamics and separation at liquidliquid interfaces. Soft Matter, 9(22):5438-5447, 2013.

[45] D. Dendukuri and P. S. Doyle. The synthesis and assembly of polymeric microparticles using microfluidics. Adv. Mater., 21(41):4071-4086, 2009.

[46] H. C. Shum, A. R. Abate, D. Lee, A. R. Studart, B. Wang, C-H. Chen, J. Thiele, R. K. Shah, A. Krummel, and D. A. Weitz. Droplet microfluidics for fabrication of non-spherical particles. Macromol. Rapid Commun., 31(2):108-118, 2010.

[47] R. McGorty, J. Fung, D. Kaz, and V. N. Manoharan. Colloidal self-assembly at an interface. Mater. Today, 13(6):34-42, 2010.

[48] Y. Lin, H. Skaff, T. Emrick, A. D. Dinsmore, and T. P. Russell. Nanoparticle assembly and transport at liquid-liquid interfaces. Science, 299(5604):226-229, 2003.

[49] G-R. Yi, T. Thorsen, V. N. Manoharan, M-J. Hwang, S-J. Jeon, D. J. Pine, S. R. Quake, and S-M. Yang. Generation of uniform colloidal assemblies in soft microfluidic devices. Adv. Mater., 15(15):1300-1304, 2003.

[50] Z. Nie, W. Li, M. Seo, S. Xu, and E. Kumacheva. Janus and ternary particles generated by microfluidic synthesis: design, synthesis, and self-assembly. J. Am. Chem. Soc., 128(29):94089412, 2006.

[51] T. M. Ruhland, A. H. Groschel, N. Ballard, T. S. Skelhon, A. Walther, A. H. E. Muller, and S. A. F. Bon. Influence of janus particle shape on their interfacial behavior at liquid-liquid interfaces. Langmuir, 29(5):1388-1394, 2013.

[52] A. Khademhosseini, M. H. May, and M. V. Sefton. Conformal coating of mammalian cells immobilized onto magnetically driven beads. Tissue Eng., 11(11-12):1797-1806, 2005.

[53] S. S. H. Tsai, J. S. Wexler, J. Wan, and H. A. Stone. Microfluidic ultralow interfacial tensiometry with magnetic particles. Lab Chip, 13(1):119-125, 2013.

[54] T. T. Perkins, D. E. Smith, and S. Chu. Single polymer dynamics in an elongational flow. Science, 276(5321):2016-2021, 1997.

[55] F. J. Galindo-Rosales, M. A. Alves, and M. S. N. Oliveira. Microdevices for extensional rheometry of low viscosity elastic liquids: a review. Microfluid. Nanofluidics, 14(1-2):1-19, 2013.

[56] S. J. Haward, A. Jaishankar, M. S. N. Oliveira, M. A. Alves, and G. H. McKinley. Extensional flow of hyaluronic acid solutions in an optimized microfluidic cross-slot devicea). Biomicrofluidics, 7(4):044108, 2013.

[57] C. de Loubens, J. Deschamps, M. Georgelin, A. Charrier, F. Edwards-Levy, and M. Leonetti. Mechanical characterization of cross-linked serum albumin microcapsules. Soft Matter, 10(25):4561$4568,2014$. 
[58] J. Soulages, M. S. N. Oliveira, P. C. Sousa, M. A. Alves, and G. H. McKinley. Investigating the stability of viscoelastic stagnation flows in T-shaped microchannels. J. Nonnewton. Fluid. Mech., 163(1):9-24, 2009.

[59] M. Tanyeri, E. M. Johnson-Chavarria, and C. M. Schroeder. Hydrodynamic trap for single particles and cells. Appl. Phys. Lett., 96(22):224101, 2010.

[60] Y. Xia and G. M. Whitesides. Soft lithography. Annu. Rev. Mater. Sci., 28(1):153-184, 1998.

[61] B-U Moon, S. G. Jones, D. K. Hwang, and S. S. H. Tsai. Microfluidic generation of aqueous two-phase system (ATPS) droplets by controlled pulsating inlet pressures. Lab Chip, 2015.

[62] Michael D Abràmoff, Paulo J Magalhães, and Sunanda J Ram. Image processing with imagej. Biophotonics international, 11(7):36-42, 2004.

[63] V. Sharma, S. J. Haward, J. Serdy, B. Keshavarz, A. Soderlund, P. Threlfall-Holmes, and G. H. McKinley. The rheology of aqueous solutions of ethyl hydroxy-ethyl cellulose (EHEC) and its hydrophobically modified analogue (mEHEC): extensional flow response in capillary break-up, jetting (ROJER) and in a cross-slot extensional rheometer. Soft Matter, 11(16):3251-3270, 2015.

[64] A. S. Geller, S. H. Lee, and L. G. Leal. The creeping motion of a spherical particle normal to a deformable interface. J. Fluid Mech., 169:27-69, 1986.

[65] J. W. J. De Folter, V. W. A. De Villeneuve, D. G. A. L. Aarts, and H. N. W. Lekkerkerker. Rigid sphere transport through a colloidal gas-liquid interface. New J. Phys., 12(2):023013, 2010.

[66] A. M. J. Shapiro, J. R. T. Lakey, E. A. Ryan, G. S. Korbutt, E. Toth, G. L. Warnock, N. M. Kneteman, and R. V. Rajotte. Islet transplantation in seven patients with type 1 diabetes mellitus using a glucocorticoid-free immunosuppressive regimen. New Engl. J. Med., 343(4):230-238, 2000.

[67] T. Wang, J. Adcock, W. Kühtreiber, D. Qiang, K. J. Salleng, I. Trenary, and P. Williams. Successful allotransplantation of encapsulated islets in pancreatectomized canines for diabetic management without the use of immunosuppression. Transplantation, 85(3):331-337, 2008.

[68] H. Walter and G. Johansson. Methods in Enzymology, volume 228. Academic Press New York, 1994.

[69] J. A. Asenjo and B. A. Andrews. Aqueous two-phase systems for protein separation: a perspective. J. Chromatogr. A, 1218(49):8826-8835, 2011.

[70] H. Walter, G. Johansson, and D. E. Brooks. Partitioning in aqueous two-phase systems: recent results. Anal. Biochem., 197(1):1-18, 1991.

[71] J. C. Merchuk, B. A. Andrews, and J. A Asenjo. Aqueous two-phase systems for protein separation: Studies on phase inversion. J. Chromatogr. B Biomed. Sci. Appl., 711(1):285-293, 1998. 
[72] U. Sivars and F. Tjerneld. Mechanisms of phase behaviour and protein partitioning in detergent/polymer aqueous two-phase systems for purification of integral membrane proteins. Biochim. Biophys. Acta, 1474(2):133-146, 2000.

[73] A. Kumar, M. Kamihira, I. Y. Galaev, B. Mattiasson, and S. Iijima. Type-specific separation of animal cells in aqueous two-phase systems using antibody conjugates with temperature-sensitive polymers. Biotechnol. Bioeng., 75(5):570-580, 2001.

[74] M. Yamada, V. Kasim, M. Nakashima, J. Edahiro, and M. Seki. Continuous cell partitioning using an aqueous two-phase flow system in microfluidic devices. Biotechnol. Bioeng., 88(4):489-494, 2004.

[75] J. P. Frampton, D. Lai, H. Sriram, and S. Takayama. Precisely targeted delivery of cells and biomolecules within microchannels using aqueous two-phase systems. Biomed. Microdevices, 13(6):1043-1051, 2011.

[76] T. Yaguchi, S. Lee, W. S. Choi, D. Kim, T. Kim, R. J. Mitchell, and S. Takayama. Micropatterning bacterial suspensions using aqueous two phase systems. Analyst, 135(11):2848-2852, 2010.

[77] C. Kepka, J. Rhodin, R. Lemmens, F. Tjerneld, and P-E. Gustavsson. Extraction of plasmid dna from escherichia coli cell lysate in a thermoseparating aqueous two-phase system. J. Chromatogr. A, 1024(1):95-104, 2004.

[78] A. M. Azevedo, P. A. J. Rosa, I. F. Ferreira, A. M. M. O. Pisco, J. De Vries, R. Korporaal, T. J. Visser, and M. R. Aires-Barros. Affinity-enhanced purification of human antibodies by aqueous two-phase extraction. Sep. Purif. Technol., 65(1):31-39, 2009.

[79] H. Song, D. L. Chen, and R. F. Ismagilov. Reactions in droplets in microfluidic channels. Angew. Chem. Int. Ed., 45(44):7336-7356, 2006.

[80] S-Y. Teh, R. Lin, L-H. Hung, and A. P. Lee. Droplet microfluidics. Lab Chip, 8(2):198-220, 2008.

[81] S. Sharma, M. Srisa-Art, St. Scott, A. Asthana, and A. Cass. Droplet-based microfluidics. Methods Mol. Biol., 949:207-230, 2013.

[82] D. K. Hwang, D. Dendukuri, and P. S. Doyle. Microfluidic-based synthesis of non-spherical magnetic hydrogel microparticles. Lab Chip, 8(10):1640-1647, 2008.

[83] M. T. Guo, A. Rotem, J. A. Heyman, and D. A. Weitz. Droplet microfluidics for high-throughput biological assays. Lab Chip, 12(12):2146-2155, 2012.

[84] L. Mazutis, J. Gilbert, W. L. Ung, D. A. Weitz, A. D. Griffiths, and J. A. Heyman. Single-cell analysis and sorting using droplet-based microfluidics. Nat. Protoc., 8(5):870-891, 2013.

[85] S. Hardt and T. Hahn. Microfluidics with aqueous two-phase systems. Lab Chip, 12(3):434-442, 2012. 
[86] R. J. Meagher, Y. K. Light, and A. K. Singh. Rapid, continuous purification of proteins in a microfluidic device using genetically-engineered partition tags. Lab Chip, 8(4):527-532, 2008.

[87] K. Vijayakumar, S. Gulati, A. J. deMello, and J. B. Edel. Rapid cell extraction in aqueous twophase microdroplet systems. Chem. Sci., 1(4):447-452, 2010.

[88] S. D. Geschiere, I. Ziemecka, V. van Steijn, G. J. M. Koper, J. H. van Esch, and M. T. Kreutzer. Slow growth of the rayleigh-plateau instability in aqueous two phase systems. Biomicrofluidics, 6(2):022007, 2012.

[89] Y. S. Song and Y. H. Choi. Microextraction in a tetrabutylammonium bromide/ammonium sulfate aqueous two-phase system and electrohydrodynamic generation of a micro-droplet. J. Chromatogr. A, 1162(2):180-186, 2007.

[90] Y. H. Choi and Y. S. Song. Droplet-based microextraction in the aqueous two-phase system. J. Chromatogr. A, 1217(24):3723-3728, 2010.

[91] I. Ziemecka, V. van Steijn, G. J. M. Koper, M. Rosso, A. M. Brizard, J. H. van Esch, and M. T. Kreutzer. Monodisperse hydrogel microspheres by forced droplet formation in aqueous two-phase systems. Lab Chip, 11(4):620-624, 2011.

[92] I. Ziemecka, V. van Steijn, G. J. M. Koper, M. T. Kreutzer, and J. H. van Esch. All-aqueous core-shell droplets produced in a microfluidic device. Soft Matter, 7(21):9878-9880, 2011.

[93] D. Lai, J. P. Frampton, H. Sriram, and S. Takayama. Rounded multi-level microchannels with orifices made in one exposure enable aqueous two-phase system droplet microfluidics. Lab Chip, 11(20):3551-3554, 2011.

[94] H. C. Shum, J. Varnell, and D. A. Weitz. Microfluidic fabrication of water-in-water (w/w) jets and emulsions. Biomicrofluidics, 6(1):012808, 2012.

[95] A. Sauret and H. C. Shum. Forced generation of simple and double emulsions in all-aqueous systems. App. Phys. Lett., 100(15):154106, 2012.

[96] Y. Song and H. C. Shum. Monodisperse w/w/w double emulsion induced by phase separation. Langmuir, 28(33):12054-12059, 2012.

[97] H. Tavana, B. Mosadegh, and S. Takayama. Polymeric aqueous biphasic systems for noncontact cell printing on cells: engineering heterocellular embryonic stem cell niches. Adv. Mater., 22(24):2628-2631, 2010.

[98] D. Forciniti, C. K. Hall, and M. R. Kula. Interfacial tension of polyethyleneglycol-dextran-water systems: influence of temperature and polymer molecular weight. J. Biotechnol., 16(3):279-296, 1990. 
[99] D. Dendukuri, Shelley S. Gu, D. C. Pregibon, T. A. Hatton, and P. S. Doyle. Stop-flow lithography in a microfluidic device. Lab Chip, 7(7):818-828, 2007.

[100] D. M. Fries, F. Trachsel, and P. R. von Rohr. Segmented gas-liquid flow characterization in rectangular microchannels. Int. J. Multiphase Flow, 34(12):1108-1118, 2008.

[101] L. Shui, A. van den Berg, and J. C. T. Eijkel. Capillary instability, squeezing, and shearing in head-on microfluidic devices. J. Appl. Phys., 106(12):124305, 2009.

[102] M. Grad, C. C. Tsai, M. Yu, D. L. Kwong, C. W. Wong, and D. Attinger. Transient sensing of liquid films in microfluidic channels with optofluidic microresonators. Meas. Sci. Technol., 21(7):075204, 2010 .

[103] T. Thorsen, S. J. Maerkl, and S. R. Quake. Microfluidic large-scale integration. Science, 298(5593):580-584, 2002.

[104] W. Li, E. W. K. Young, M. Seo, Z. Nie, P. Garstecki, C. A. Simmons, and E. Kumacheva. Simultaneous generation of droplets with different dimensions in parallel integrated microfluidic droplet generators. Soft Matter, 4(2):258-262, 2008.

[105] B-U. Moon, S. S. H. Tsai, and D. K. Hwang. Rotary polymer micromachines: in situ fabrication of microgear components in microchannels. Microfluid. Nanofluidics, pages DOI: 10.1007/s10404015-1548-6, 2015.

[106] H. Dong, W. W. Carr, and J. F. Morris. An experimental study of drop-on-demand drop formation. Phys. Fluid., 18(7):072102, 2006.

[107] J-C. Galas, D. Bartolo, and V. Studer. Active connectors for microfluidic drops on demand. New J. Phys., 11(7):075027, 2009.

[108] C. Berkland, K. K. Kim, and D. W. Pack. Fabrication of plg microspheres with precisely controlled and monodisperse size distributions. J. Controlled Release, 73(1):59-74, 2001. 